\title{
Vasculitis allergica - ein nicht-IgE-vermitteltes Hypersensitivitätssyndrom
}

\author{
Vasculitis allergica - a non-IgE-mediated \\ hypersensitivity syndrome
}

Autoren

S. Lutze, W. Konschake, M. Ahmed, A. Arnold, T. Westphal, H. Riebe, G. Daeschlein, M. Jünger

Institute

Klinik und Poliklinik für Hautkrankheiten,

Universitätsmedizin Greifswald

Schlüsselwörter

Vaskulitis, Vasculitis allergica, leukozytoklastische Vaskulitis (LCV), Immunkomplex-Erkrankungen, kutane IgM oder IgG Immunkomplex-Vaskulitis

Key words

vasculitis, vasculitis allergica, leukocytoclastic vasculitis (LcV), immune complex diseases, cutaneous IgM or lgC immune complex vasculitis

eingereicht 27.02.2018

akzeptiert 29.01.2019

Bibliografie

DOI https://doi.org/10.1055/a-0847-6602

Online-Publikation: 10.05.2019

Phlebologie 2019; 48: 251-265

(c) Georg Thieme Verlag KG Stuttgart · New York

ISSN 0939-978X

Korrespondenzadresse

Dr. med. Stine Lutze

Klinik und Poliklinik für Hautkrankheiten

Universitätsmedizin Greifswald

F.-Sauerbruch Straße

17489 Greifswald

Tel. + 493834866770

E-Mail: stine.lutze@uni-greifswald.de

Englische Version unter:

https://doi.org/10.1055/a-0847-6602

\section{ZUSAMMENFASSUNG}

Die Diagnose „Vasculitis allergica“ suggeriert eine klassisch allergische IgE-vermittelte Genese. Dies ist jedoch nicht der Fall, vielmehr handelt es sich um einen hochkomplex ablaufenden Mechanismus, der häufig das eigentlich auslösende Antigen vor der Detektion bewahrt.

Die aktuelle Nomenklatur für dieses dermatologische Erkrankungsbild lautet kutane IgM oder lgG Immunkomplex-Vaskulitis, es handelt sich um eine leukozytoklastische Vaskulitis der postkapillären Venolen [3]. Diese neue Einordnung legt die mit dem ursprünglichen Namen einhergehende Assoziation mit einer typisch IgE-vermittelten allergischen Erkrankung ab.

Vaskulitiden werden immunologisch betrachtet den Immunkomplex-Erkrankungen, der sogenannten Typ III Reaktion nach Coombs und Gell, zugeordnet [14, 15, 16]. Sie werden als entsprechend dem dahinter liegenden immunologischen Prozess als „non-IgE-mediated allergic hypersensitivity Syndrom“ eingestuft $[14,15,16]$. Viele Vaskulitiden machen sich klinisch zuerst an der Haut u. a. durch akut auftretende, rasch progrediente Ulzerationen bemerkbar, häufig assoziiert mit starker Schmerzhaftigkeit. Sie sind damit in der Ursachenabklärung eines Ulcus cruris bedeutsam, rangieren hier aber unter den seltenen Diagnosen.

Der lange bestehende Begriff einer „leukozytoklastischen Vaskulitis“ für diese Erkrankung steht entsprechend nicht länger für ein einzelnes Erkrankungsbild, sondern vielmehr für ein histologisches Muster einer Gruppe von Vaskulitiden, den sogenannten Immunkomplexvaskulitiden. [3] Hierbei handelt es sich von Gefäßkaliber überwiegend um „small vessel“ Vaskulitiden, diese können als „single organ vasculitis“ (SOV) mit alleiniger Manifestation an der Haut oder auch als kutanes Teilsymptom einer anderen „multi organ vasculitis“ (MOV) zum Beispiel im Rahmen eines systemischen Lupus erythematodes auftreten [1]. Die kutane IgM oder IgG Immunkomplex-Vaskulitis zeigt den für Vaskulitiden klassisch plötzlich auftretenden, rasch progredienten Verlauf und ist klinisch durch eine typische Primäreffloreszenz, die palpable Purpura, mit hoher Inflammation charakterisiert. [2] Im Therapiekonzept steht an erster Stelle das auslösende Agens zu identifizieren und zu eliminieren [2, 42, 44]. In der Regel zeigt dieser Typ von Vaskulitis eine überwiegend residuenfreie Abheilung mit niedrigem Rezidivrisiko unter der Voraussetzung, dass das auslösende Agens identifiziert und beseitigt ist $[2,41,42,44]$.

\section{ABSTRACT}

The diagnosis "Vasculitis allergica" suggests a classical allergic IgE-mediated genesis. However, this is not the case; rather, it is a highly complex mechanism that often prevents the antigen that actually triggers the antigen from being detected.

The current nomenclature for this dermatological disease pattern is cutaneous IgM or lgG immunocomplex vasculitis, which 
is leukocytoclastic vasculitis of the postcapillary venules [3]. This new classification discards the association associated with the original name with a typically lgE-mediated allergic disease. Vasculitides are immunologically associated with immune complex diseases, the so-called type III reaction according to Coombs and Gell $[14,15,16]$. They are classified as "non-IgE-mediated allergic hypersensitivity syndrome" according to the underlying immunological process $[14,15,16]$. Many vasculitides are clinically first noticeable on the skin, e. $g$. by acute, rapidly progressive ulcerations, often associated with severe pain. They are therefore important in the clarification of the causes of venous leg ulcers, but here they rank among the rare diagnoses.

The long-standing term "leukocytoclastic vasculitis" for this disease no longer stands for a single disease pattern but rather for a histological pattern of a group of vasculitides, the socalled immune complex vasculitides [3]. Most of the vascular calibres are small vessel vasculitis, which can occur as single organ vasculitis (SOV) with sole manifestation on the skin or as a cutaneous partial symptom of another multi organ vasculitis (MOV), for example in the context of systemic lupus erythematosus [1]. Cutaneous IgM or IgG immunocomplex vasculitis shows the classic sudden, rapidly progressive course for vasculitis and is clinically characterized by a typical primary fluorescence, the palpable purpura, with high inflammation [2]. In the therapy concept, the first priority is to identify and eliminate the triggering agent $[2,42,44]$. Usually this type of vasculitis shows a predominantly residueless healing with low risk of recurrence, provided that the triggering agent is identified and eliminated $[2,41,42,44]$.
Klassifikation der Vaskulitiden als Erkrankungsgruppe und Einordnung der kutanen IgM oder IgG-Immunkomplex-

\section{Vaskulitis}

Die in 2012 überarbeitete international geltende Chapel Hill Consensus Conference (CHCC) Nomenklatur der Vaskulitiden zeigt die Mannigfaltigkeit dieser Erkrankungsgruppe und macht deutlich, wie wichtig hierbei die Anamnese und die genaue klinische Deskription für die Diagnosefindung ist. In die CHCC- Klassifikation flossen die Ätiologie, die Pathogenese, das Kaliber des Gefäßes, der Entzündungstyp, die hauptsächlich betroffenen Organe mit klinischer Manifestation, die genetische Prädisposition und demografische Parameter mit ein. Durch die 2012 erarbeitete Revision wird die Erkrankungsgruppe der Vaskulitiden in der Darstellung übersichtlicher, erfährt eine Spezifikation und erleichtert den Umgang mit dem Krankheitsbild im klinischen Alltag [1]. In 2018 wird ein dermatologisches Addendum zu dieser bestehenden CHCCKlassifikation von 2012 ergänzt. Ziel dieses Zusatzes war es, eine einheitliche Nomenklatur und Definition der kutanen Vaskulitiden festzulegen [3].

Der erste Kategorisierungslevel bezieht sich weiterhin auf das Gefäßkaliber, welches von der Entzündung betroffen ist. Es findet eine Unterteilung in große Gefäße, mittelgroße Gefäße und kleine Gefäße statt [1]. Daneben existiert eine weitere Gruppe, die Vaskulitis der variablen Gefäße, welche jedes Gefäß-Kaliber betrifft [1].

Neben dieser Größenzuordnung wird in der CHCC 2012 eine sogenannte „Ein-Organ-Vaskulitis“ als Begriff neu implementiert, wobei per Definition nur ein Organ von der Vaskulitis ergriffen wird. Daneben existiert eine Gruppe Vaskulitiden, die mit Systemerkrankungen assoziiert ist und eine Gruppe, deren Vertreter an eine spezifische Ätiologie gebunden ist $[1,4,7,11]$.

Der lange für das Krankheitsbild der kutanen IgM oder lgG Immunkomplex-Vaskulitis verwendete Begriff der leukozytoklastischen Vaskulitis (LCV) spiegelt letztlich das histologische Bild einer Vaskulitis-Untergruppe wieder und steht im dermatologischen Addendum der CHCC-Klassifikation als Synonym für diese Untergrup- pe, den sogenannten Immunkomplex-Vaskulitiden, der häufigsten Form der Vaskulitis mit einer kutanen Manifestation [3, 11].

Die kutane IgM oder lgG Immunkomplex-Vaskulitis zeigt dementsprechend histologisch das Muster der leukozytoklastischen Vaskulitis in der Haut und wird der Kleingefäßvaskulitis (small vessel vasculitis - SVV) zugeordnet und zunächst als „single organ vasculitis“ (SOV) mit alleiniger Manifestation an der Haut eingestuft. Ob dies zukünftig Bestand hat oder auch im Rahmen dieses Vaskulitis-Typs andere Organsysteme im Sinne einer MOV tangiert sind, werden entsprechende klinische Beobachtungen zeigen [1, 3, 7, 8, 9, 11].

\section{Vaskulitiden der kleinen Gefäße (small vessel vasculitis - SVV)}

\section{Ein-Organ-Vaskulitis (single organ vasculitis - SOV)}

Hierbei stellt die IgM oder IgG Immunkomplex-Vaskulitis den wohl bedeutsamsten Vertreter der Gruppe der SVV dar. Die kutane leukozytoklastische Vaskulitis (LCV, in der aktuellen Literatur der am häufigsten verwendete Begriff, der dieses Erkrankungsbild beschreibt) ist mit einer Inzidenz von 10-20/100 000 eine der häufigsten Vaskulitiden überhaupt [1, 3, 19, 20, 21, 44].

Die sich leukozytoklastisch manifestierende Vaskulitis kann daneben als Teilsymptom einer Systemerkrankung auftreten [5].

\section{Mit Systemerkrankungen assoziierte leukozytoklastische Vaskulitis}

Diese, häufig auch als sekundäre Vaskulitiden bezeichnet, treten zum Beispiel klassisch im Rahmen von Kollagenosen auf, vor allem die typische Lupusvaskulitis der Haut aber auch die Ulcera bei einer systemischen Sklerodermie [5]. Ulzerationen im Rahmen von Kollagenosen zeigen das typische Verlaufsmuster und den klinischen Verlauf einer Vaskulitis, sie sind hochakut und stark entzündlich und beginnen häufig unter dem Bild einer klassischen LcV ( und sind mit starken Schmerzen verbunden (Ischämie-Schmerz). 


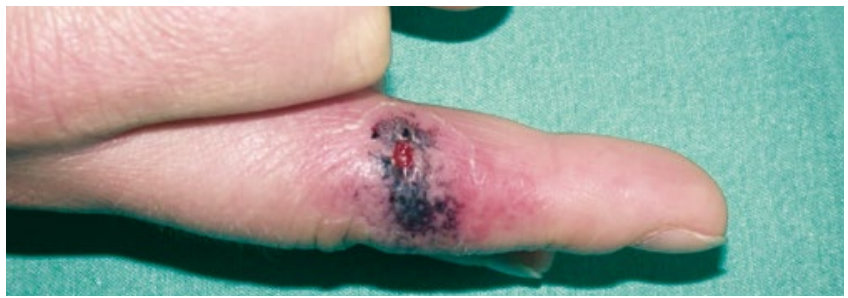

- Abb. 1 Kutan manifestierte Vaskulitis mit akralen Ulcera als Komponente einer systemischen Vaskulitis im Rahmen eines SLE
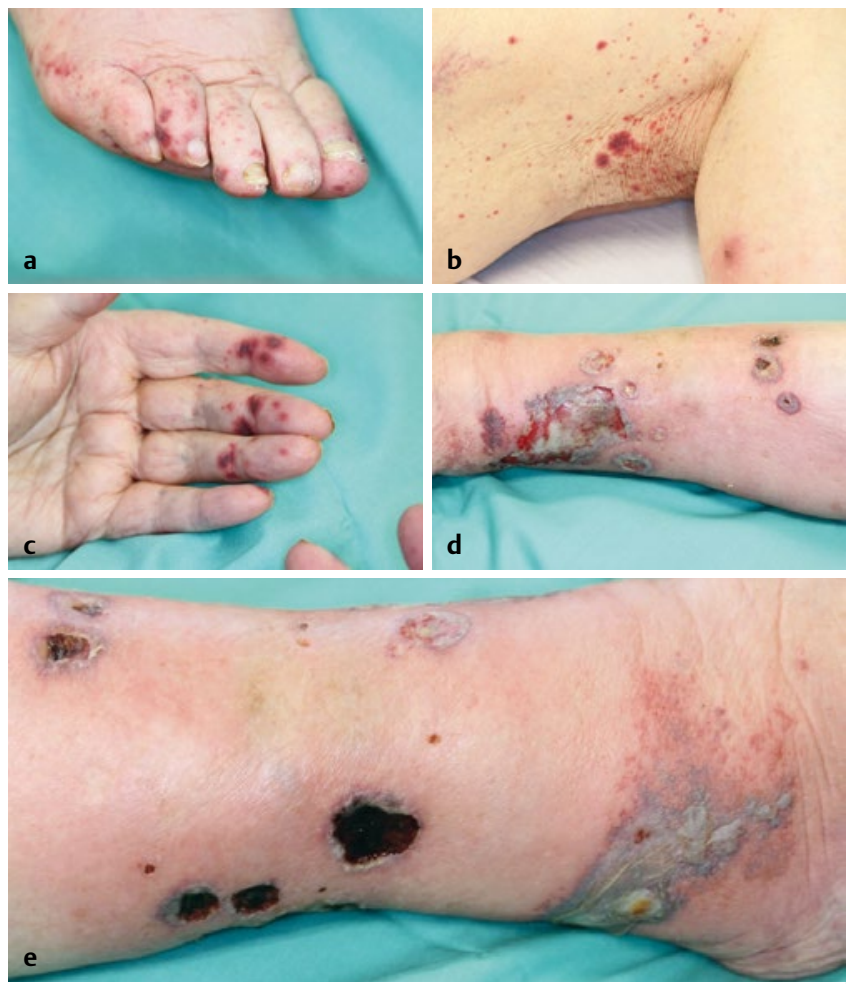

- Abb. 2 a Kasus 1 „Torasemid-Aufdosierung und akutes Nierenversagen“: bei Erstvorstellung zeigen sich einzelne Effloreszenzen an den Zehen; $\mathbf{b}$ Kasus 1: 5 Stunden später besteht eine disseminierte Aussaat von palpablen Petechien an den Beinen auf den Rumpf übergehend; c Kasus 1: Im weiteren Verlauf zeigten sich palpable Purpura auch an den Händen; d Kasus 1: Etwa 24 Stunden später zeigten sich erste Epidermolysen, rasch gefolgt von Ulzerationen; e Kasus 1: Weitere 24 Stunden später zeigten sich festhaftende Nekrosen.

\section{Kasuistische Darstellung des Krankheitsbildes}

Exemplarisch zur Veranschaulichung des klinischen Verlaufes und der ursächlichen Zusammenhänge bei der „Erfüllung“ des pathophysiologischen Mechanismus einer IgM oder IgG ImmunkomplexVaskulitis erfolgt innerhalb des Manuskriptes die kasuistische Darstellung von 2 typischen Erkrankungsverläufen. Als Verweis darauf sind diese Passagen „kursiv“ hinterlegt.

Im ersten Fall handelte es sich um eine 97-jährige multimorbide Patientin. Im Rahmen einer klinisch beobachteten Zunahme von Unterschenkelödemen bei bekannter Herz- und Niereninsuffizienz wurde hier eine Diuretika-Therapie mit Torasemid $10 \mathrm{mg} / \mathrm{d}$ be-
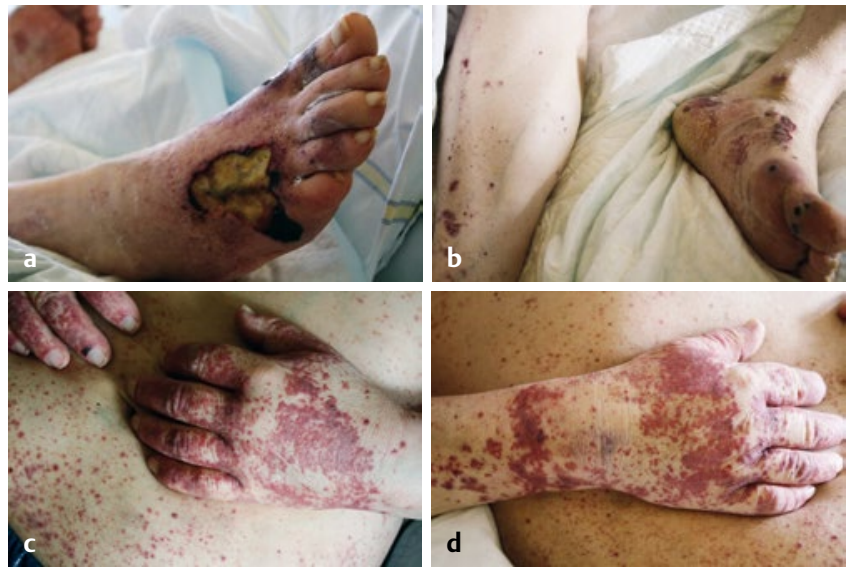

Torasemid $2 \times 10 \mathrm{mg} / \mathrm{d}$

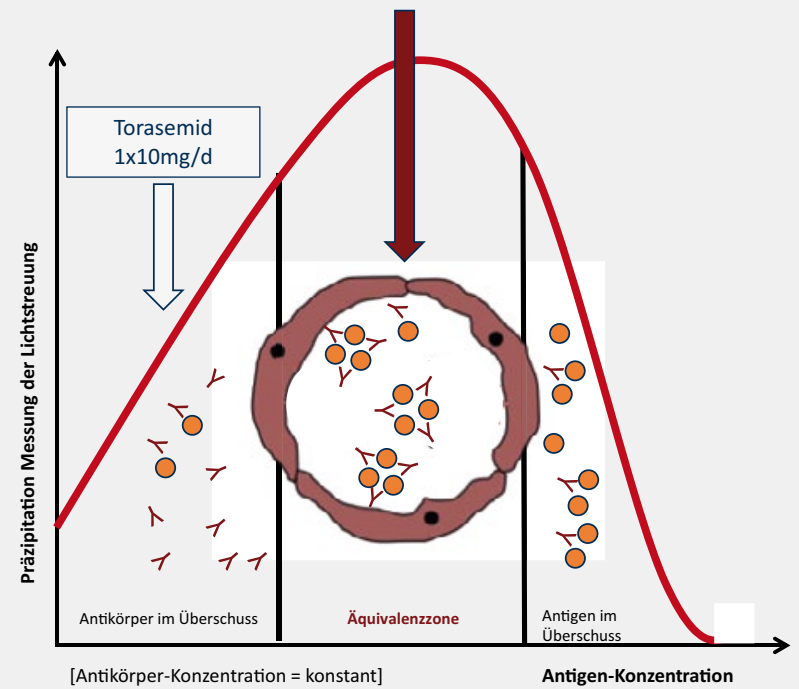

Präzipitatkurve - Heidelberger Kurve

Abb. 3 a-b Kasus 2 - konsiliarische Erst-Vorstellung eines Patienten über die Kardiologische Station mit ausgedehnten NekroseZonen am rechten ( $\bullet \mathbf{A b b} \mathbf{3} \mathbf{3 a})$ und linken ( $\bullet \mathbf{A b} \mathbf{b} . \mathbf{3 b})$ Fuß nach stattgehabter kutaner IgM oder IgG Immunkomplex-Vaskulitis a. e. unter neu aufdosiertem Torasemid bei Herzinsuffizienz mit 2x10mg; c-d Kasus 2 erneuter fulminanter Schub der Vaskulitis (c: linke Hand; d: rechte Hand) bei erneuter Torasemid-Aufdosierung auf $2 \times 10 \mathrm{mg}$ nach vorheriger kompletter Pausierung mit darunter kompletter Remission des Befundes; e Erreichen der Äquivalenzzone mit klinisch Ausbildung von vaskulitischen Herden bei einer Dosis von 2x10mg Torasemid.

gonnen. Bei unzureichender Besserung erfolgte 5 Tage nach Initiierung eine Aufdosierung auf $2 \times 10 \mathrm{mg} / \mathrm{d}$, weitere 5 Tage später traten erste petechiale Hautveränderungen an den Zehen auf ( $\boldsymbol{A} \boldsymbol{A} \boldsymbol{b} \boldsymbol{b} . \mathbf{2 a}$ ), es zeigte sich dann ein rasch progredienter Hautbefund, der zu einer Akutvorstellung in der Ambulanz der Hauklinik der UMG führte, hier verschlechterte sich sowohl der Hautbefund als auch der Gesamtzustand der Patientin stündlich $(\boldsymbol{A} \boldsymbol{b} \boldsymbol{b}$. $2 \mathbf{b}-\nabla \boldsymbol{A} \boldsymbol{b} \boldsymbol{b} . \mathbf{2 e}$ ). Bei dem 2. Kasus handelte es sich um einen 65jährigen Patienten. Hier bestanden bei konsiliarischer Erstvorstellung über die kardiologische Station seit 6 Wochen ausgedehnte vaskulitisch veränderte Flächen am gesamten Integument mit einhergehender Nekrotisierung an den Füßen und Un- 


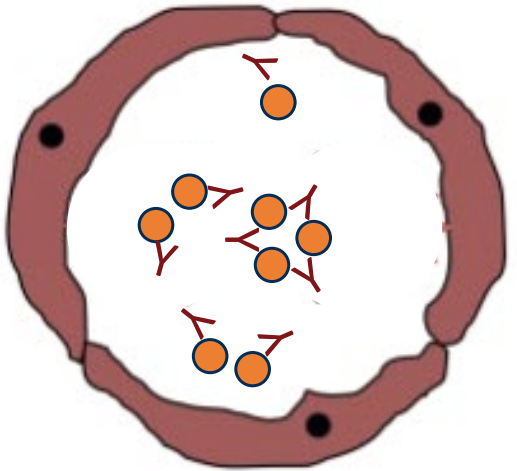

Formation von AG-AK-Immunkomplexen

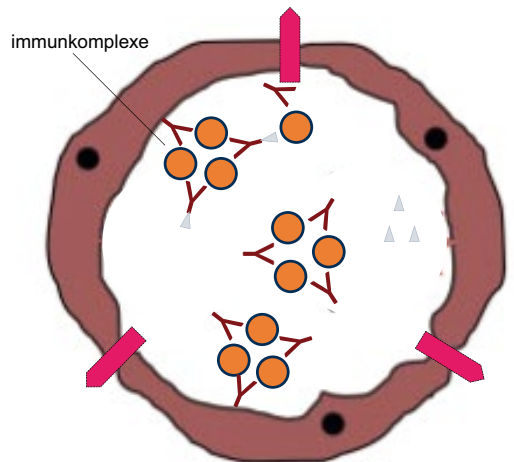

Zirkulierende Immunkomplexe binden an die Gefäßwände und werden in die Basalmembran eingeschlossen.

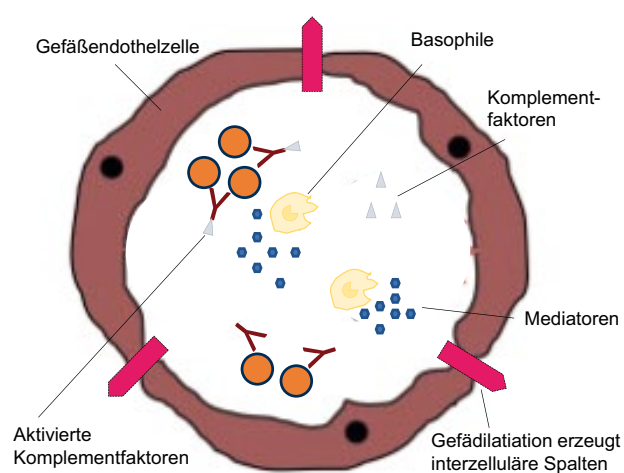

Die entstandenen Immunkomplexe aktivieren über den klassischen Aktivierungsweg das Komplementsystem. Dies führt zur Degranulation der Basophilen, zahlreiche Mediatoren werden freigesetzt, welche unter anderem die

b Gefäßperaeabilität steigern.

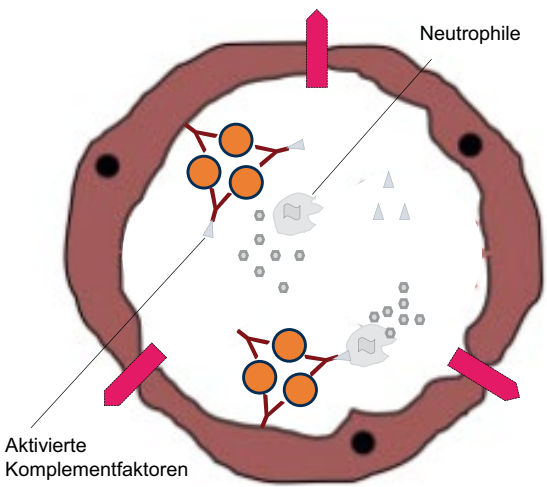

Komplementfaktoren ziehen Neutrophile an und lösen deren Degranulation aus.

-Abb. 4 a-e Typ III Reaktion nach Coombs und Gell - Immunkomplex-Reaktion - Vaskulitis

terschenkeln ( $\boldsymbol{A} \boldsymbol{A} \boldsymbol{b} \boldsymbol{b} . \mathbf{3} \mathbf{a}-\boldsymbol{A} \boldsymbol{b} \boldsymbol{b} . \mathbf{3} \boldsymbol{b})$. Anamnestisch fand 5 Tage vor Auftreten der ersten Hautveränderungen eine Aufdosierung von Torasemid von ursprünglich langjährig $10 \mathrm{mg}$ auf $45 \mathrm{mg}$ statt bei kardialer Dekompensation.

\section{Immunologischer Mechanismus der Immunkomplex- Reaktion in Korrelation zur Klinik und Histologie mit entsprechendem $A b$ - und Verlauf}

Die frühere Bezeichnung der IgM oder IgG Immunkomplex-Vaskulitis als Vasculitis allergica geht auf eine irrtümliche Interpretation der Genese der Erkrankung zurück. Vaskulitiden werden immuno- logisch als Typ III Reaktion nach Coombs und Gell eingeordnet. Dies beinhaltet einen Antigen-Antikörper-Aggregat-Mechanismus, der den Begriff der Immunkomplex-Erkrankung geprägt hat. Es wird immunologisch als sogenanntes non-IgE-mediated allergic hypersensitivity Syndrom eingestuft. Es handelt sich um eine humoral vermittelte Immunreaktion durch IgM oder IgG getragen $[3,13$, $14,15,16,45]$.

Auch bei den Typ III Reaktionen kann es ähnlich der Typ I Reaktion zu einem Sensibilisierungsphänomen kommen, welches im Verlauf bei Re-Exposition auch zu Rezidiven führen kann. Die Latenz- 


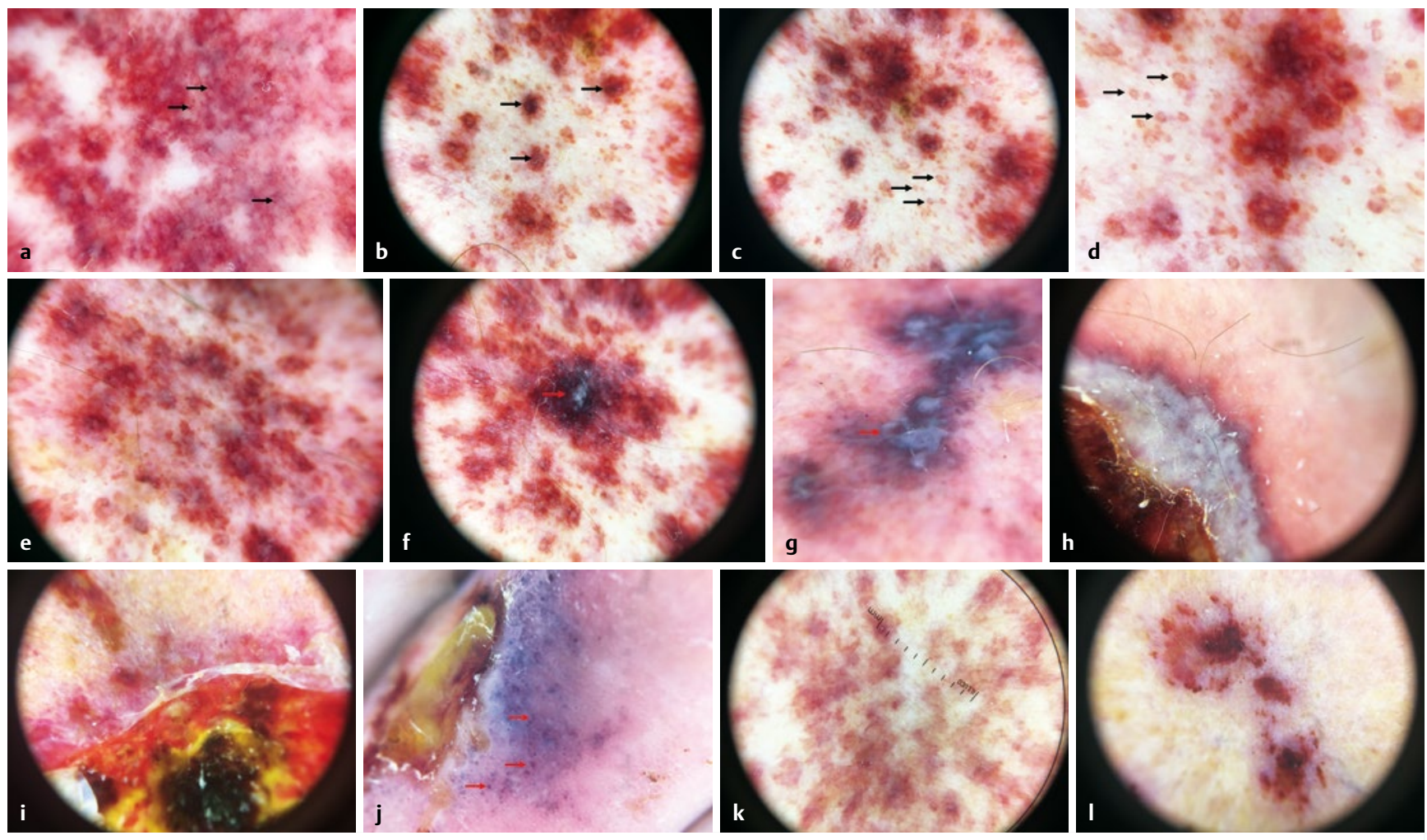

Abb. 5 a Dermatoskopie Vollbild der kutanen IgM oder IgG Immunkomplex-Vaskulitis: in den Effloreszenzen werden zentral stehende prominente Gefäße als Dots und Globules (Pfeile) sichtbar mit umgebendem hämorrhagisch-purpurisch fleckigem unscharfem Hintergrund (= typisch für ein vaskulitisches Geschehen). Der Hintergrund korreliert klinisch mit dem livid-erythematösen Grundton ( $\mathbf{A} \mathbf{b b}$. 7a-b) und entspricht histologisch den Erythrozytenextravasaten ( $\mathbf{A} \mathbf{b b} \mathbf{b} \mathbf{6 b})$; b Dermatoskopie Vollbild der kutanen IgM oder IgG Immunkomplex-Vaskulitis: Mit dem Fortschreiten des Entzündungsprozesses nimmt das perivaskuläre Infiltrat und Ödem und die Erythrozytenextravasation zu, dies entspricht der klinisch bestehenden palpablen Purpura (Pfeile) ( $\mathbf{A b b}$. 7a-b); c-d Dermatoskopie Vollbild der kutanen IgM oder IgG Immunkomplex-Vaskulitis: typisch sind die Aussparungen der Follikel sichtbar als perifollikuläre purpurischen Halo-Figuren (Pfeile); e Dermatoskopie Vollbild der kutanen IgM oder IgG Immunkomplex-Vaskulitis: im weiteren Verlauf der Erkrankung beginnen die Einzeleffloreszenzen, ineinander überzugehen und zu großen Flächen zu konfluieren; f Dermatoskopie Vollbild der kutanen IgM oder IgG Immunkomplex-Vaskulitis: im weiteren Verlauf der Erkrankung zeigen sich im Zentrum der palpablen Purpura erste blau-weiß schimmernde Areale, die histologisch beginnenden Epidermolysen entsprechen und klinisch Vesiculae (Pfeil); g Dermatoskopie Vollbild der kutanen IgM oder lgG Immunkomplex-Vaskulitis: im weiteren Verlauf der Erkrankung konfluieren die blau-weiß schimmernden Areale zu klinisch Bullae. (Pfeil); h Dermatoskopie Vollbild der kutanen IgM oder IgG ImmunkomplexVaskulitis: im weiteren Verlauf der Erkrankung konfluieren die blau-weiß schimmernden Bullae zu großen Flächen; i Dermatoskopie: Vollbild der kutanen IgM oder IgG Immunkomplex-Vaskulitis: im weiteren Verlauf der Erkrankung entstehen festhaftende Nekrosen und Ulzerationen auf Grund der Mangelversorgung der Haut bei vaskulitisch verursacht thrombotisch verschlossenen Gefäßen in der papillären Dermis; j Dermatoskopie: Vaskulitisch verursachtes Ulcus: es zeigt sich ein Ulcus mit hochinflammatorisch imponierendem Randsaum, blau-weiß schimmernder Umgebungshaut durchsetzt mit Dots und Globules (Pfeile) in lividem Hintergrund als Nachweis einer weiter aktiven Vaskulitis; kDermatoskopie: in Abheilung befindliche kutane IgM oder IgG Immunkomplex-Vaskulitis, es zeigen sich keine Dots und Globules mehr als Zeichen einer aktiven Vaskulitis. Klinisch ist die palpable Purpura nicht mehr nachweisbar. Der bestehende livid-erythematöse Hintergrund (Hämorrhagie) bildet sich über gelb-braune fleckige Muster (Abbau der Erythrozyten) zurück; I Dermatoskopie: in Abheilung befindliche kutane IgM oder IgG Immunkomplex-Vaskulitis, die Vesiculae und Bullae heilen über hämorrhagischen Krusten ab.

zeit bis zur Reaktion nach Erstexposition beträgt durchschnittlich 8-13 Tage, bei Re-Exposition 6-12 Stunden [47].

Der im Rahmen einer Immunkomplexreaktion ablaufende Mechanismus beginnt mit der Diffusion von Allergen-spezifischen IgGund IgM -Antikörper aus den Blutgefäßen ins Gewebe, hier bilden sie mit dem spezifischen Allergen Immunkomplexe, diese lagern sich an der Basalmembran von Gefäßendothelien ab ( $\vee$ Abb. 4a) und können in Gewebeschnitten mittels einer direkten Immunfluoreszenz nachgewiesen werden, wichtig hierbei zur Abgrenzung von der IgA-positiven LcV ist der Nachweis der Immunglobulin-Subtypen IgG und IgM als Teil des Immunkomplexes [3, 5, 13, 14, 15 , 16]. Klinisch zeigen sich erste petechial anmutende leicht erhabene stecknadelkopfgroße Papeln als Korrelat des histologisch nach- weisbaren ektasierten, wandverdickten Gefäßes (i. d. R. postkapilläre Venolen, aber auch kleine Venen und Arteriolen) $[3,6,7,8$, 18, 20, 21, 42, 44] ( A Abb. 5a, > Abb. 6a). Dermatoskopisch zeigen sich die ektasierten wandverdickten Gefäße (Vasodilatation) als tief düsterrote, teils livide Dots und Globules $[46,48,49]$ ( $\mathbf{A b b}$. 5a). Die ersten Läsionen erscheinen für das Krankheitsbild typisch an den Füßen und Unterschenkeln. Dies ist vermutlich ein Stase (Vasodilatation) bedingter Effekt. Die Gefäßweitstellung fördert die Ablagerung von Immunkomplexen [2, 3, 21].

In der Folge kommt es durch die abgelagerten Immunkomplexe zu einer Aktivierung des Komplementsystems, insbesondere der Komplementfaktoren C3a und C5a. Komplementbeladene Immunkomplexe aktivieren über die Bindung an verschiedenen Zellrezep- 

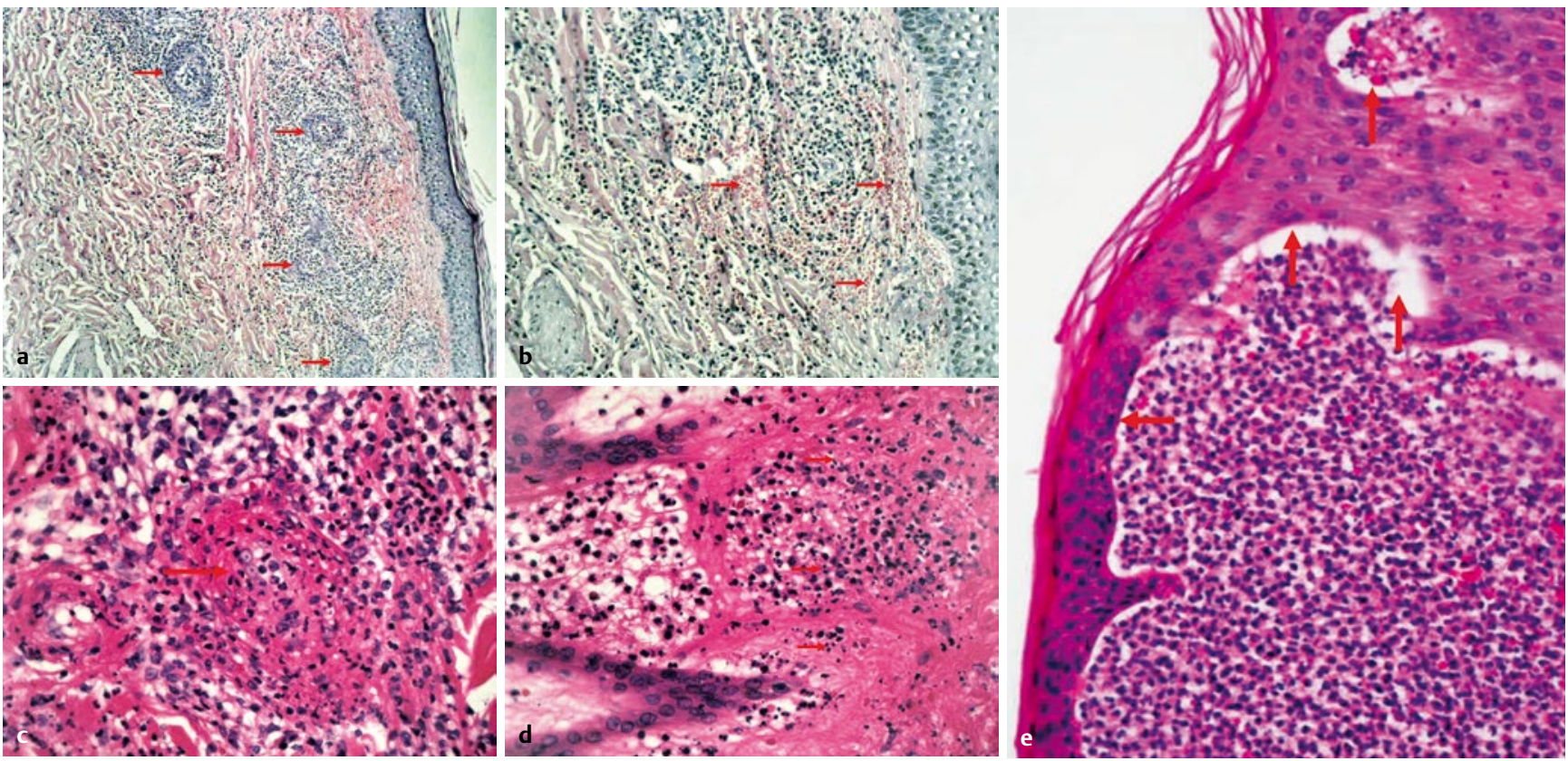

\begin{abstract}
Abb. 6 a Histologisches Vollbild einer kutanen leukozytoklastischen Vaskulitis mit ektasierten wandverdickten Gefäßen (Pfeile) im papillaren Plexus mit umgebenden granulozytär-lymphozytären Infiltraten (HE-Färbung, Vergrößerung 10x/0,25); b Histologisches Vollbild einer kutanen leukozytoklastischen Vaskulitis mit ektasierten wandverdickten Gefäßen im papillaren Plexus mit umgebenden granulozytär-lymphozytären Infiltraten und kräftig Erythrozytenextravasaten (Pfeile) (HE-Färbung, Vergrößerung 20x/0,40); c Histologisches Vollbild einer kutanen leukozytoklastischen Vaskulitis mit wandverdicktem, thrombosiertem Gefäß (Pfeil) im papillaren Plexus mit umgebenden granulozytär-lymphozytären Infiltraten (HE-Färbung, Vergrößerung $20 \times / 0,40$ ); d Sichtbarer Kernstaub als Hinweis auf die stattgefundene frustran verlaufene Phagozytose mit resultierender Leukozytoklasie (Pfeile) (HE-Färbung, Vergrößerung $40 \times / 0,65$ ); e Histologie einer fortgeschrittenen kutanen leukozytoklastischen Vaskulitis mit ektasierten wandverdickten Gefäßen im papillaren Plexus mit umgebenden granulozytär-lymphozytären Infiltraten, Kernstaub und kräftig Erythrozytenextravasaten. Zusätzlich zeigen sich subepidermale Spaltbildungen (Pfeile) und granulozytäre Infiltrate durchsetzt mit Erythrozyten und kräftig Kernstaub. Dieser Befund entspricht klinisch den Epidermolysen in Form von hämorrhagischen Vesikulae und Bullae.

( Abb. 5f, $>$ Abb.5g, $>$ Abb. 7c, > Abb. 7d, ) (HE-Färbung, Vergrößerung 20x/0,40)
\end{abstract}

toren von in den Blutgefäßen zirkulierenden Immunzellen (Fc-Rezeptoren von Mastzellen, Makrophagen, Granulozyten, T-Lymphozyten) ( $\mathbf{A} \mathbf{A b} \mathbf{b} \mathbf{4} \mathbf{b}, \triangleright \mathbf{A b b} \mathbf{4} \mathbf{4} \mathbf{c})$. Nach stattgehabter frustraner Teil-Phagozytose (die Immunkomplexe sind zu groß, um komplett phagozytiert zu werden) der Immunkomplexe kommt es zur Freisetzung einer Vielzahl von Mediatoren (Leukotriene, Prostaglandine und anderen Zytokinen) mit resultierender weiterer Zunahme der Inflammation in den Gefäßwänden ( $>$ Abb.4b->Abb.4d). Die Gefäße werden im Rahmen der Mediator-getriggerten Vasodilatation zunehmend permeabel, es treten Plasmaproteine ins Gewebe über, die Inflammation breitet sich perivaskulär aus $[3,5,13,14,15$, 16] ( $\triangleright$ Abb. 4b- $\mathbf{A b b . 4 d ) . ~ H i s t o l o g i s c h ~ z e i g e n ~ s i c h ~ n u n ~ d e u t l i c h ~}$ wandverdickte kleine Gefäße im Stratum papillare der Dermis umgeben von einem granulozytär dominierten Infiltrat durchmischt mit Lymphozyten, Eosinophilen und deutlich sichtbaren Erythrozytenextravasaten als indirekter Hinweis auf die destruierten Venolen des papillaren Plexus ( $>$ Abb. $\mathbf{6 b}$, $\triangleright \mathbf{A b b} \mathbf{6} \mathbf{6 c}$ ). Klassisch kommen nun auch die „zerfallenden“ Granulozyten (Leukozytoklasie) mit sichtbarem Kernstaub („Kerntrümmer“) zur Darstellung, die letztlich den Namen des Erkrankungsbildes als „leukozytoklastische Vasculitis“ geprägt haben [3, 6, 7, 8, 18, 20, 21, 42, 44] (• Abb. 6d). Dieses Fortschreiten der Erkrankung wird klinisch an der Zunahme der inflammatorischen Komponente der Einzeleffloreszenzen sichtbar. Um die palpablen Petechien tauchen nun hämorrhagische Höfe auf mit einer zentrifugalen Ausdehnung ( $\triangleright$ Abb. 7a- $\mathbf{A b b}$. 7b). Auflichtmikroskopisch ist nun das Vollbild einer LcV erreicht, neben den zentralen Dots bzw. Globules zeigen sich die hämorrhagischen Höfe, die ineinander konfluieren und den typischen tief livid-erythematös fleckigen, verschwommenem Hintergrund bilden. Das fleckige Muster ist typisch für eine vaskulitische Genese der purpurischen Veränderungen ( $\mathbf{A} \mathbf{b} \mathbf{b} . \mathbf{5 a} \mathbf{\text { a }} \mathbf{A} \mathbf{b} \mathbf{b} . \mathbf{5 b}$ ). Typisch sind auch die perifollikulären purpurischen Halo-Figuren [46, 48, 49] ( A Abb. 5c, \Abb. 5d). Die Veränderungen sind mechanisch nicht wegdrückbar, da es sich um Einblutungen und thromboembolisch verschlossene Gefäße (Dots, Globules) handelt. Der klinische Rumpel-Leede-Test (Auslösen von Petechien nach Druckaufbau auf $10 \mathrm{mmHg}$ mittels einer Blutdruckmanschette über einen Zeitraum von 5 Minuten) ist positiv als Nachweis der erhöhten Fragilität der entzündeten Gefäße.

Pathophysiologisch haben die Granulozyten und Makrophagen angefangen, die Immunkomplexe zu phagozytieren. Auf Grund der Größe der Immunkomplexe verläuft diese Phagozytose frustran, phagozytierende Zellen gehen unter, dies macht die histologisch sichtbare Leukozytoklasie im Gewebe aus [3, 18, 21] (• Abb. 6d). Andere Immunzellen diffundieren in noch offene Gefäße und transportieren Immunkomplexe in die Milz und Leber als klassische Abbauorgane der Immunkomplexe. Dieser Mechanismus kann sich bei entsprechender Menge an Immunkomplexen bzw. dem Vorliegen anderer Co-Faktoren erschöpfen, dieser Zustand liegt bei einer klinisch manifesten Vaskulitis vor $[13,14,15,16]$.

Schreitet dieser immunologische Prozess nun weiter fort, kommt es zum Untergang des epidermalen Gewebes auf Grund 

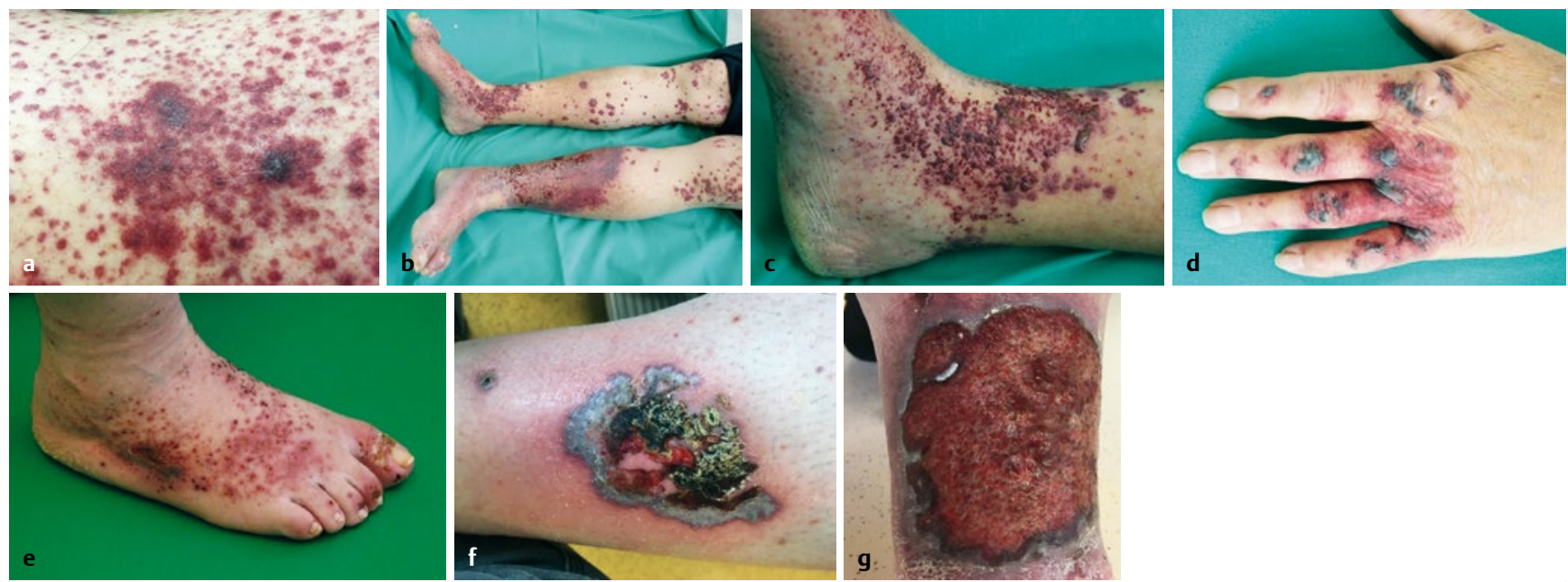

- Abb. 7 Klinisches Vollbild der kutanen IgM oder IgG Immunkomplex-Vaskulitis mit einer zentral erhabener Purpura (palpabel) und umgebendem hochinflammatorisch- hämorrhagischen Hof (a Detailaufnahme; b Übersichtsaufnahme); c Klinisches Vollbild der kutanen IgM oder IgC Immunkomplex-Vaskulitis, mit Fortschreiten der Erkrankung zeigen sich Epidermolysen in Form von hämorrhagischen Vesikeln und Bullae am Fuß; d Klinisch Vollbild der kutanen IgM oder IgG Immunkomplex-Vaskulitis mit Fortschreiten der Erkrankung zeigen sich Epidermolysen in Form von hämorrhagischen Vesikeln und Bullae an der Hand; e Klinisches Vollbild der kutanen IgM oder IgG Immunkomplex-Vaskulitis mit Fortschreiten der Erkrankung zeigen sich Epidermolysen in Form von hämorrhagischen Vesikeln und Bullae neben den Beinen im weiteren Verlauf auch an den Händen; $\mathbf{f U ̈ b e r g a n g ~ d e r ~ V e s i k e l ~ u n d ~ B u l l a e ~ i n ~ U l z e r a t i o n e n ~ m i t ~ z u n a ̈ c h s t ~ f e s t h a f t e n d e n ~ N e k r o s e n ~ u n d ~ h o c h i n f l a m m a t o r i s c h e n ~ R a ̈ n d e r n ~ a l s ~}$ Ausdruck des Fortschreitens der Vaskulitis; g Ulcus cruris, vasculitischer Genese mit einem düsterroten Randsaum.

der fehlenden nutritiven Versorgung bei vaskulitisch-thrombotisch verschlossenen Gefäßen. Klinisch zeigen sich nun zunächst Epidermolysen in Form von Vesikeln und Bullae, die rasch in Nekrosen (ischämischer Untergang des Gewebes) übergehen. [2, 20, 21, 44] ( Abb. 6e, $>$ Abb. 7c $-\triangleright$ Abb. 7g) Dermatoskopisch zeigen sich diese Veränderungen als weiß-blau schimmernde Patches ( $\bullet$ Abb. 5f, $>$ Abb. 5g). Dieser Vorgang ist primär auf die Primäreffloreszenz das palpable Petechien beschränkt, im Verlauf dehnt er sich in die Peripherie sich aus, bizarr konfiguriert, z. T. Blitzfigurenartig mit hochinflammatorischen Rändern bei hier weiter aktivem und fortschreitendem Prozess der Vaskulitis. ( $\mathbf{A b b}$. 5e, $\vee$ Abb. $\mathbf{5 h}$, - Abb. 7f) Das Vollbild der Erkrankung zeigt klassisch ein Nebeneinander von frischen Effloreszenzen, neben Nekrosen und Ulzerationen und sich in Abheilung befindlichen Arealen, die gelb-bräunlich (Siderophagen) abklingen ( $\boldsymbol{A} \mathbf{A b b} . \mathbf{5 k}, \triangleright \mathbf{A b b} \mathbf{5 I} \mathbf{l})$.

Die Läsionen beginnen in der Regel an den Füßen und Unterschenkeln und z. T. Händen, steigen aber bei fehlender Intervention rasch über die Oberschenkel zum Stamm auf, es gibt auch einzelne Fallberichte mit Beteiligung des Gesichtes [2, 20, 21, 44] ( Abb. 8).

Entscheidend für das Entstehen von großen pathogenen Immunkomplexen ist die jeweilige Menge Antigen und Antikörper: Wenn beide in ähnlich hoher Konzentration vorliegen, ist die sogenannte Äquivalenzzone erreicht, die Antigen-Antikörper-Komplexe (Präzipitate) fallen in großer Anzahl aus. Wenn eine der beiden Komplex-Bestandteile überwiegt fallen nur wenig und kleine Komplexe aus. Dieses Phänomen ist sehr bedeutsam, es verdeutlicht, dass z. B. oft erst die Dosis-Änderung oder ein gestörter Abbau eines Medikamentes, im Falle einer Medikamentenassoziation, das Krankheitsbild auslöst und eine Änderung es auch kausal behandelt [47] ( Abb. 9).

Die eigentliche Pathogenität der Immunkomplexe ist abhängig von der Löslichkeit, der Ladung und dem Ort der Ablagerung (u. a.

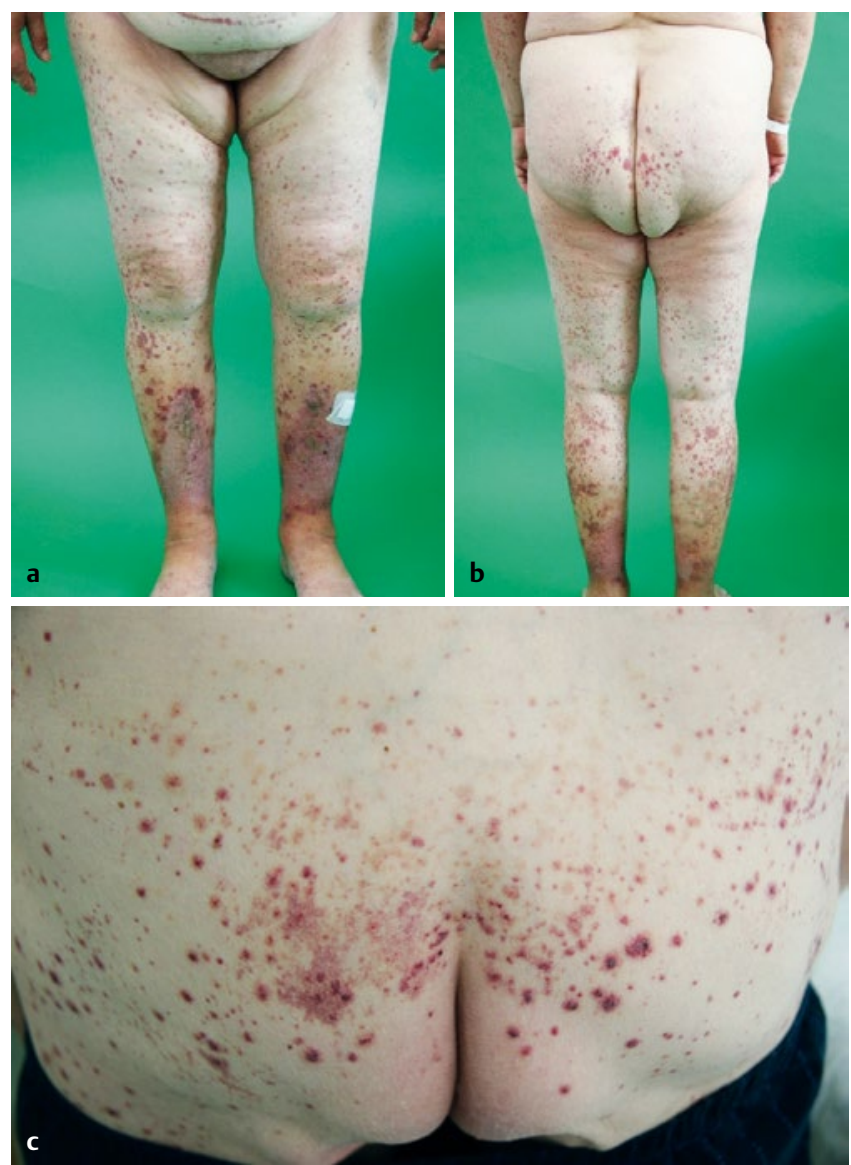

Abb. 8 a-c Klinisches Verteilungsmuster der kutanen IgM oder IgG Immunkomplex-Vaskulitis mit Beginn an den Füßen und Unterschenkeln und Übergang auf den Körperstamm. 


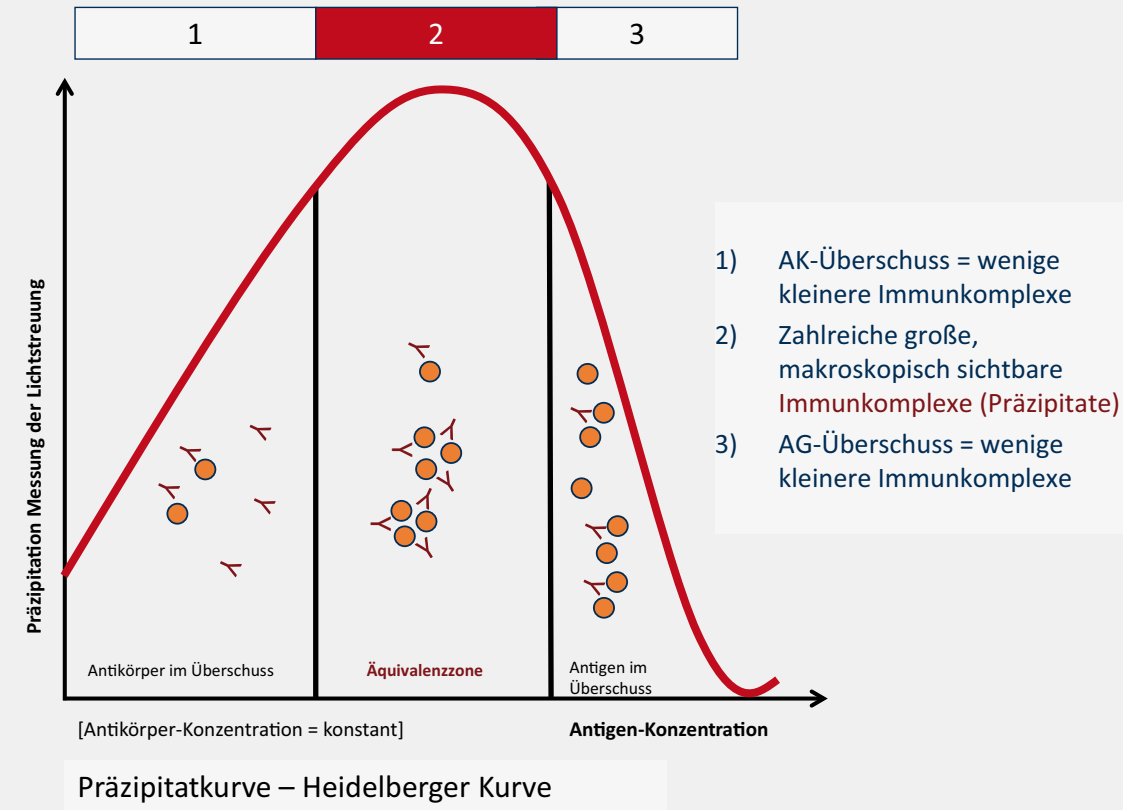

Abb.9 Präzipitatkurve der Typ III Reaktion nach Coombs und Gell, sogenannte Heidelberger Kurve.

Haut, Niere). Der eigentliche pathogene Prozess setzt ein, wenn die Eliminationskapazität der Milz bzw. Leber erschöpft ist, erst dann kommt es zu einer klinisch wirksamen Ablagerung der Immunkomplexe in der Niere, der Haut, den Gelenken, Muskeln, der Lunge und im Gehirn. Dabei lagern sich positiv geladene Immunkomplexe bevorzugt an negativ geladenen Basalmembranzonen der Haut und der Glomeruli ablagern. Unlösliche Immunkomplexe wirken im Gewebe wenig pathogen, während partiell lösliche zu den vorbeschriebenen lokalen Entzündungsreaktionen führen. Bei der IgM oder lgG Immunkomplex-Vaskulitis als SOV ist in der Regel nur die Haut betroffen, da sich IgM und IgG kaum im Mesangium der Glomeruli ablagert, beide werden wesentlich schneller abgebaut als IgA, welches typisch die IgA-vermittelte Nephritis verursacht im Rahmen einer IgA positiven Vaskulitis [2, 47].

Das fortgeschrittene Stadium der Erkrankung zeigt vaskulitische Ulzera an den Beinen, diese haben einen raschen Progress, sind geprägt von hochinflammatorischen Rändern, erreichen schnell eine eindrucksvolle Tiefe und werden von starker Schmerzhaftigkeit begleitet durch den raschen ischämischen Gewebeuntergang und die hohe Gewebeinflammation im Rahmen der Freisetzung von Entzündungsmediatoren ( $\triangleright$ Abb. 7f, $>$ Abb.7g, $\triangleright$ Abb. 5i, $>$ Abb. 5j).

\section{Diagnosestellung}

Die Diagnose einer IgM oder lgG Immunkomplex-Vaskulitis wird in erster Linie anhand der klassischen Klinik, der Anamnese, des Verlaufes, der Histologie und Immunhistologie und sekundär durch entsprechend negative Autoantikörperserologien (ANA, ANCA) und hiermit Ausschluss anderer Kleingefäß-Vaskulitiden gestellt [2, 3, 4, 7, 8, 12, 42, 44].

Im Rahmen der Diagnose einer Vaskulitis ist es immer bedeutsam ob es sich um eine SOV handelt oder eine Sekundär-Vaskulitis im Rahmen einer Systemerkrankung, z. B. aus dem Kreis der Kol- lagenosen. Diese Fragestellung hat großen Einfluss auf die Therapie und Prognose.

\section{Ätiologie/Ursachen}

Die Genese der IgM oder IgG Immunkomplex-Vaskulitis ist mannigfaltig, Infektionen und Medikamente werden als am häufigsten diskutiert aber auch eine paraneoplastische Genese ist in diesem Kontext bedeutsam. Im Rahmen einer infektiösen Genese sind es überwiegend Infekte der oberen Atemwege verursacht durch Streptokokken und Adenoviren. Weiter sollte nach Virushepatitiden (Hepatitis B und C), einer Parvovirus B19-Infektion und Enteroviren gesucht werden. Häufige auslösende Medikamente sind die NSAID, Sulfonamide, Penicilline, Cefaclor, Diuretika wie HCT oder Furosemid, Allopurinol, Chinolone, Hydralazin, MTX und auch Ovulationshemmer [2, 3, 4, 7, 8, 12, 42, 44].

Bei der Medikamentenanamnese sind nicht nur neue Medikamente zu erfragen, besonders wichtig sind Dosis-Anpassungen und andere neu aufgetretene Erkrankungen wie z. B. eine Niereninsuffizienz oder Lebererkrankung, die sowohl die Elimination von Medikamenten als auch den Abbau möglicher entstandener Immunkomplexe verzögern bzw. komplett verhindern.

Weiter sind kurzzeitige Interaktionen von Medikamenten zu betrachten (verzögerter/gesteigerter Abbau), hier speziell NSAR, die oft vom Patienten nicht erinnert werden, da es sich um klassische „Gelegenheitsmedikamente“ handelt. Die Sensibilisierungswahrscheinlichkeit ist ebenso auf Grund dieser „Gebrauchsmentalität“ von NSAR für alle Typen an allergischen Reaktionen überdurchschnittlich hoch. Eine weitere ursächlich oft unterschätzte Substanzgruppe sind die Diuretika sowohl die Thiazide als auch die Schleifendiuretika. Häufig werden diese Substanzen für den Patienten anamnestisch nicht sofort erfassbar in Kombinationspräparaten verschrieben. 
In beiden Kasuistiken konnte das Schleifendiuretikum Torasemid als auslösendes Agens identifiziert werden. In beiden Fällen ist die Substanz jedoch nicht isoliert als neue Substanz zu betrachten, in einem Fall kommt eine akute Niereninsuffizienz hinzu und in dem anderen Fall spielt die Aufdosierung des Medikamentes die entscheidende Rolle. Im ersten Fall der 97-jährigen Frau wurde bei Aufnahme serologisch ein akutes Nierenversagen nachgewiesen, „aufgepfropft“ auf das bestehende chronische Nierenversagen. Dieser Zustand ist am wahrscheinlichsten durch eine Diuretika-assoziierte Exsikkose erklärt. Die Aufdosierung der Diuretika in Kombination mit der zunehmenden eingeschränkten Nierenfunktion sind vermutlich die Auslöser der Bildung von pathologisch fungierenden Immunkomplexen bei Erreichen des Äquivalenzbereiches von Antigen (Torasemid) und Antikörper.

Auch die Kombination aus einem Infekt und einem Medikament kann ursächlich zusammen eine kutane IgM oder lgG Immunkomplex-Vaskulitis auslösen. Bis zu 50\% der Erkrankungsfälle können ursächlich nicht geklärt werden (idiopathisch) [2, 42, 44].

Das klinische Bild einer IgM oder IgG Immunkomplex-Vaskulitis muss diagnostisch zwingend hinsichtlich der Möglichkeit des Auftretens als Teilsymptom einer Autoimmunerkrankung (Lupus erythematodes) bewertet werden.

\section{Differentialdiagnosen}

Das Feld der Differentialdiagnosen für dieses Krankheitsbild ist vielfältig und kann nur exemplarisch ausgeführt werden. Bei der Betrachtung der Differentialdiagnosen ist es vorteilhaft, die beiden großen klinischen Verlaufsphasen der Erkrankung getrennt voneinander zu betrachten, d. h. es gibt die zum einen Diagnosen mit klinisch sichtbaren „palpablen Purpura“ und zum anderen Diagnosen mit „Nekrosebildung und Ulzerationen“, die ausgeschlossen werden sollten. Jedoch ist es auch hierbei ähnlich wie bei der LcV, auch bei einigen differentialdiagnostisch zu betrachtenden Krankheitsbildern zeigen sich beide klinischen Verlaufsbilder in direkter Abfolge, hier werden die Erkrankungen dem bei Erstdiagnose häufig vorliegendem Erscheinungsbild zugeordnet ( $\mathbf{A b b}$. 10).

\section{Differentialdiagnosen zum klinischen Bild der „palpablen Purpura“ (exemplarisch)}

In dieser Gruppe gibt es eine ganze Reihe vital bedrohlicher, fulminant verlaufender Erkrankungen, die eine Differenzierung enorm wichtig machen.

Der erste wohl bedeutendste Vertreter ist die IgA-positive Vaskulitis, nach den Erstbeschreibern als Purpura Schoenlein-Henoch benannt. Sie ist die häufigste Vaskulitis im Kindesalter. Sie tritt typischerweise im Alter vom 4.-7. Lebensjahr auf, Jungen sind deutlich häufiger betroffen als Mädchen. Die Inzidenz liegt bei 15-25/100 000. Daneben gibt es auch eine Variante des Erwachsenenalters, die mit einer deutlich niedrigeren Inzidenz von 3/100 000 auftritt. Unabhängig vom Alter handelt es um eine Multisystemerkrankung mit Manifestationen an der Haut, den Gelenken, der Niere, dem Darm, selten auch im ZNS und in den Hoden. Typisch ist das gehäufte Auftreten nach einem Atemwegsinfekt. Das Krankheitsbild zeigt einen schubweisen Verlauf und kann bis zu 2 Jahre anhalten. Auch danach können Rezidive auftreten. Pa-

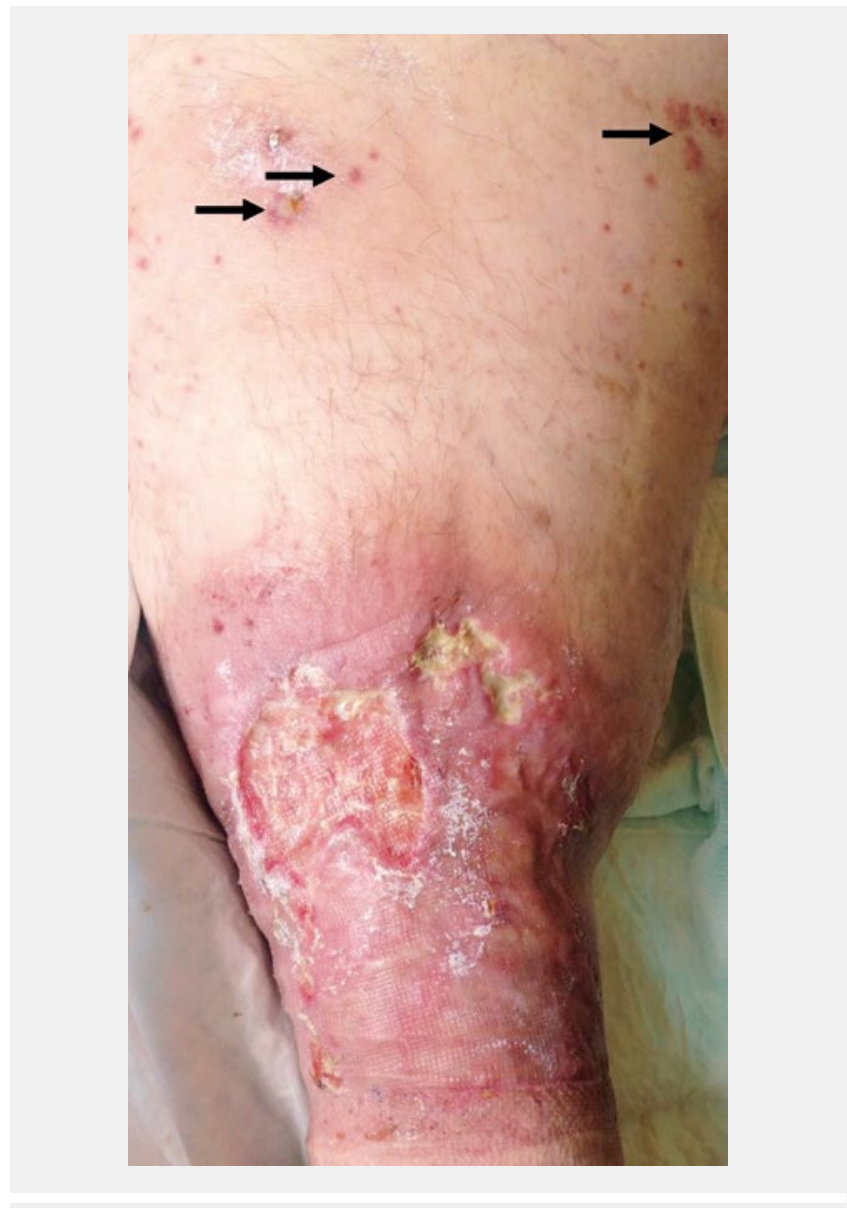

-Abb. 10 Prätibial zeigt sich ein unregelmäßig begrenztes, stark schmerzhaftes Ulcus mit umgebender Gewebeentzündung, in der Peripherie zeigen sich klassische palpable Petechien mit zentral z. T. kleinen Vesikulae als klinisch typische Manifestation einer kutanen $\mathrm{LCV}$, welche kausal für die Ulzeration verantwortlich ist.

thophysiologisch sind hier Immunglobuline vom Subtyp IgA1als Auslöser identifiziert, auch hier sind die postkapillären Venolen hauptsächlich betroffen, aber auch kleinere Venen und Arteriolen. Klinisch zeigen sich die palpablen Petechien oft netzförmig im Ausbreitungsmuster. Auch hier zeigen sich die Hautveränderungen vor allem an den Beinen. Die Erkrankung tritt auch bei Erwachsenen auf, hier zeigen sich häufig rein kutan manifestierende Verläufe $[2,10,25,26,27,29]$.

Ein weiteres Krankheitsbild, welches sich klinisch mit petechialen Veränderungen bemerkbar macht, ist die Purpura fulminans, auch als Waterhouse-Friderichsen-Syndrom bekannt. Ätiologisch handelt es sich in der Regel um eine perakut verlaufende Meningokokken-Sepsis. Auch Pneumokokken, Staphylokokken können ursächlich nachgewiesen werden. Pathologisch kommt es im Rahmen der Sepsis zu Mikrozirkulationsstörungen und begleitend zu einer disseminierten intravasalen Gerinnungsstörung (DIC) und Schock bei akut auftretender Nebenniereninsuffizienz durch hämorrhagische Infarkte. Daneben zeigen sich klassisch an der Haut zunächst petechiale Veränderungen, die rasch zunehmen und in mumifizierende Nekrosen vor allem der Akren übergehen. Die Letalität liegt bei $15-20 \%$ [40]. 

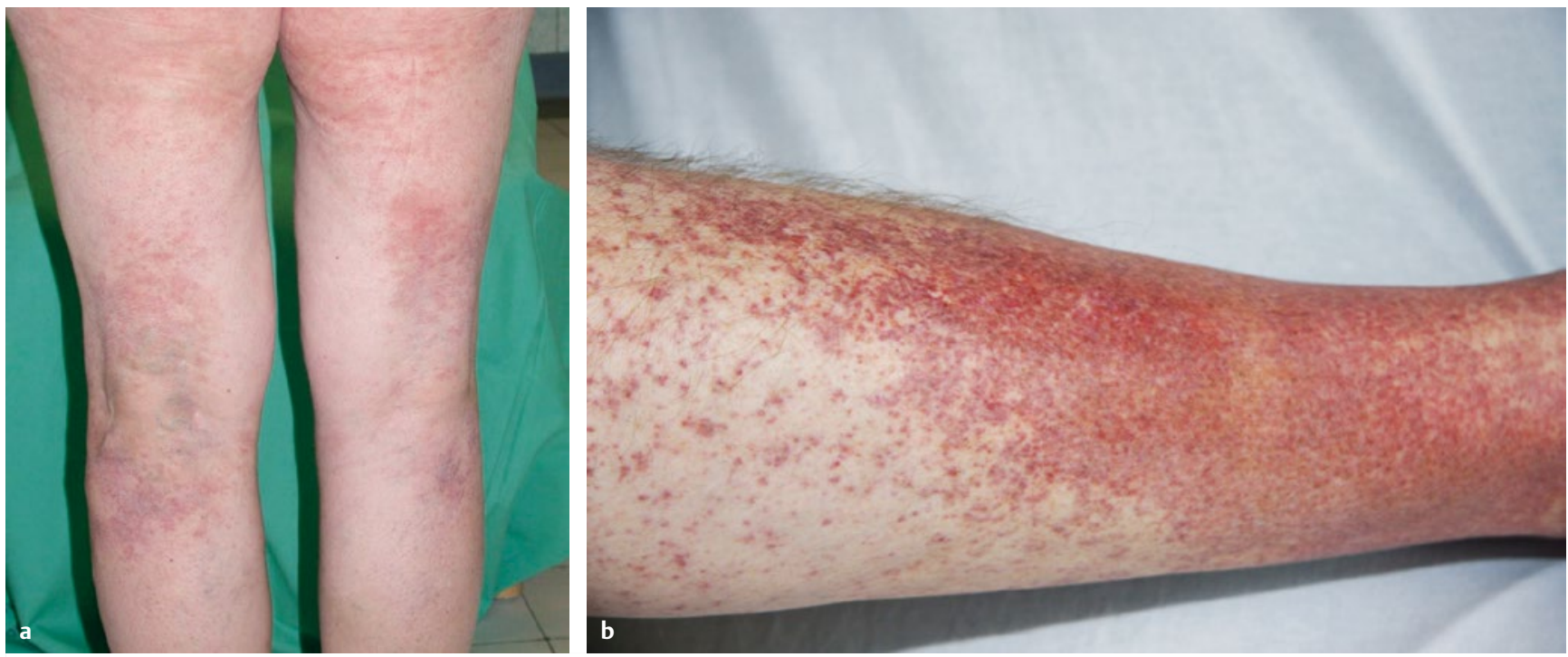

Abb. 11 a Purpura pigmentosa progressiva unter einer Langzeittherapie mit Mirtazapin; b Purpura pigmentosa progressiva unter einer Langzeittherapie mit Mirtazapin
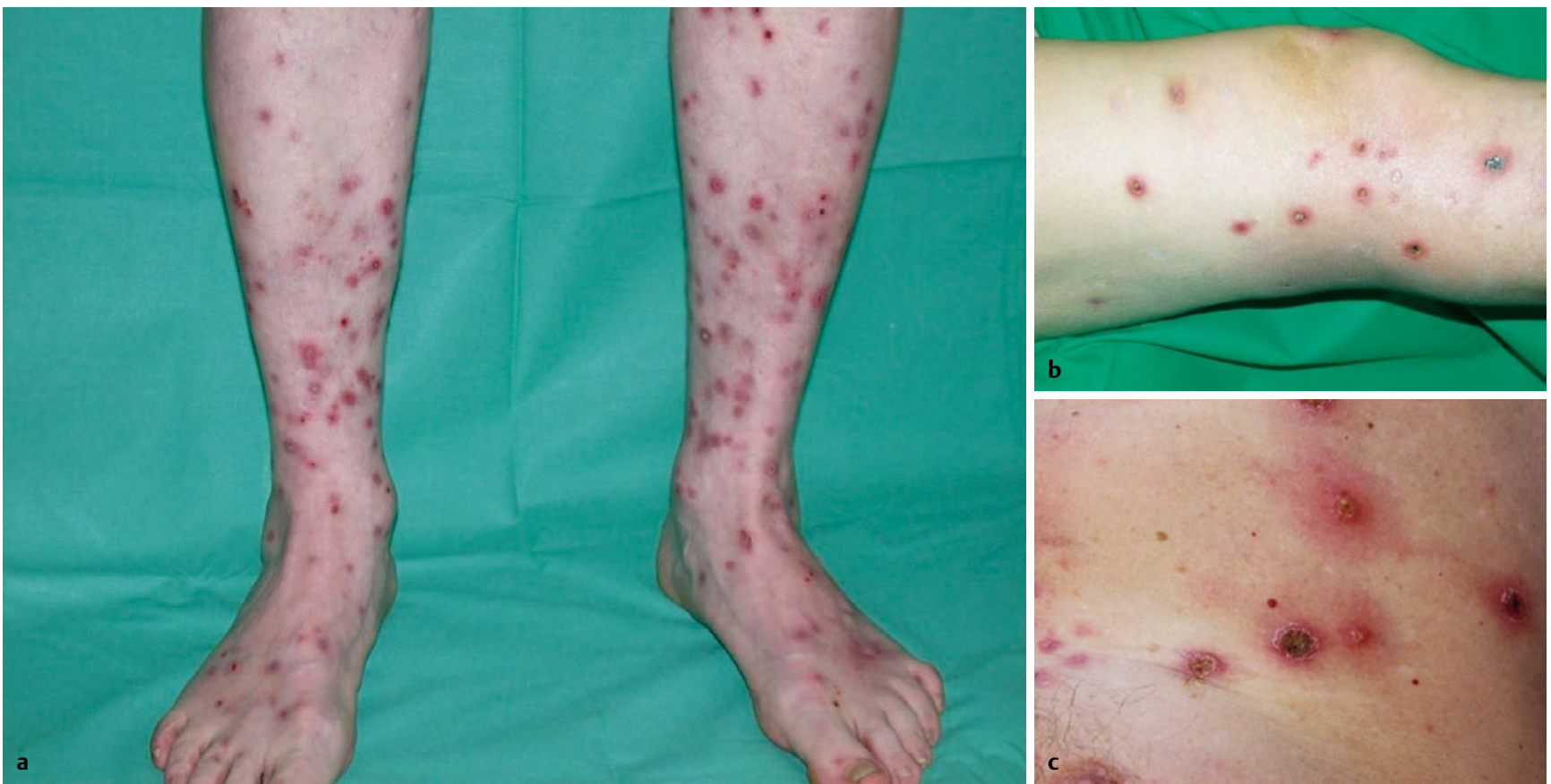

Abb. 12 Pityriasis lichenoides et varioliformis acuta (PLEVA); a an der unteren Extremität; b im Kniebereich; c im Detail pectoral

Eine weitere Differentialdiagnose für das petechiale Stadium der kutanen Immunkomplex-Vaskulitis ist die Purpura pigmentosa progressiva, der hier vermutete Entstehungsmechanismus geht mit einer Typ IV Sensibilisierung ggü. verschiedener Medikamente (u. a. Diazepame und Carbamide) einher [45, 47]. Klinisch imponiert das Erkrankungsbild durch an den Unterschenkeln, i. V. auch am gesamten Integument symmetrische, bräunlich-rote, unregelmäßige Flecken mit kleinen, cayennepfefferartigen, nicht wegdrückbaren Petechien. Wichtig ist das es sich hierbei i. d. R. um einen makulösen Befund handelt, der nicht tastbar ist ( $\mathbf{A b b}$. 11a- - Abb. 11b).

In diesem klinischen Formenkreis muss auch die Pityriasis lichenoides acuta et varioliformis Mucha-Habermann (PLEVA) als eine
Dermatose mit einer infektallergisch-bakteriellen (hämolysierende Streptokokken), medikamentös-allergische oder viralen (Herpes-zoster-Virus, Epstein-Barr-Virus) Ursache betrachtet werden $[2,21]$. Klinisch klassisch ist die sogenannte „Heubner'sche Sternkarte“, sie ist durch akut auftretende, $0,2-0,4 \mathrm{~cm}$ große, rot oder rot-braune zunächst lichenoide Papeln charakterisiert, die im Verlauf zu Erosionen und Ulzerationen mit hämorrhagischen Krusten zerfallen ( $\triangleright$ Abb. 12a- $>$ Abb. 12c).

Daneben gibt es Vielzahl weiterer Erkrankungen, die papulöse petechiale Effloreszenzen zeigen, häufig aber klinisch andere Verläufe und zusätzliche Hautveränderungen und Symptome aufweisen, die eine Differenzierung von der kutanen IgM oder IgG Immun- 


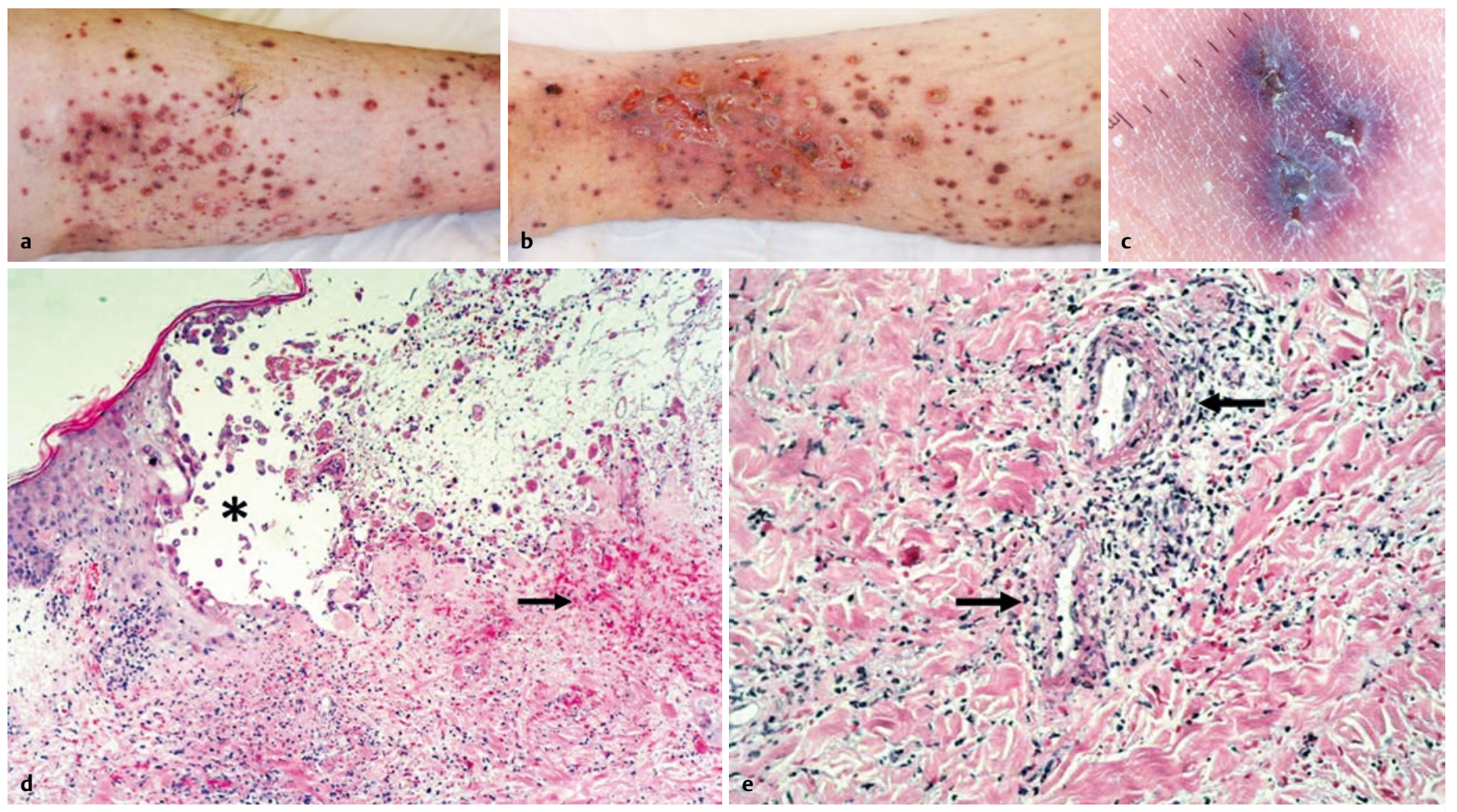

\begin{abstract}
- Abb. 13 Vaskulitisch verlaufender multisegmentaler Herpes zoster der Dermatome L4-S2 rechts (a medial; b lateral) unter laufender Immunsuppression mit Cellcept bei Z. n. Nierentransplantation; c Dermatoskopie eines vaskulitisch verlaufenden Herpes zoster: es zeigen sich die zentral genabelten Bläschen, gruppiert stehend, umgeben von hämorrhagischen Höfen. Die typischen Dots und Globules des LcV als Ausdruck der entzündlich veränderten Gefäße fehlen jedoch; d Histologie einer klinisch vaskulitisch imponierenden Herpes-Infektion der Haut mit sichtbaren intraepidermalen multilokulären Blasen, Akantholysezonen mit einzeln stehenden abgerundeten Keratinozyten $\left({ }^{*}\right)$, z. T. mehrkernigen Geisterzellen. Subepidermal zeigen sich in der Dermis kräftig Erythrozytenextravasate (Pfeil), die verantwortlich für das vaskulitisch imponierende klinische Bild ( Abb. 13a) sind. (HE-Färbung, Vergrößerung 4×/0,10); e Histologie einer klinisch vaskulitisch imponierenden Herpes-Infektion der Haut: die Gefäße der papillären Dermis stellen sich mit einem diskreten perivaskulären Infiltrat dar ohne morphologische Gefäßwandveränderungen und ohne Nachweis von Neutrophilen und entsprechender Leukozytoklasie (Pfeile) (HE-Färbung, Vergrößerung 10×/0,25).
\end{abstract}

komplex-Vaskulitis ermöglichen. So zeigt ein vaskulitisch verlaufender Herpes zoster klinisch die klassischen Effloreszenzen einer LcV und auch einen vergleichbar akuten Verlauf, die Einseitigkeit und der segmentale Befall des Befundes machen die Abgrenzung von einer LcV klinisch sicher möglich ( $\mathbf{A} \mathbf{A b} \mathbf{b} . \mathbf{1 3 a}->\mathbf{A b b}$. 13c). In der Histologie zeigt sich eine intraepidermale multilokuläre Bläschenbildung durch retikuläre Degeneration und Akantholyse. Daneben bestehen ausgedehnte Keratinozytennekrosen und mehrkernige Geisterzellen. In der oberen Dermis zeigt sich ein perivaskuläres lymphozytäres Infiltrat ohne Nachweis von Gefäßveränderung und Leukozytoklasie $[2,21]$ ( A Abb. 13d-^Abb. 13e).

\section{Differentialdiagnosen zum klinischen Bild der „Nekrosebildung mit folgender Ulzeration“}

Auch in diesem Stadium der Erkrankung gibt es eine große Vielzahl an Erkrankungen, die differentialdiagnostisch betrachtet werden müssen. Auch hier ist es vor allem die Gruppe der Vaskulitiden, speziell die Gruppe der Kleingefäß- Vaskulitiden (SVV), welche zu den wichtigsten Differentialdiagnosen zählen. Zum einen müssen die ANCA-assoziierten Vaskulitiden (AAV) betrachtet werden. Diese Erkrankungsgruppe kann klinisch primär als LcV manifest werden. Da die Diagnosestellung häufig verzögert erfolgt, zeigen sich hier oft bereits Nekrosen und Pyoderma gangraenosum-artige Ulzerationen an der Haut. Einzelne AAV zeigen rein kutane Manifestatio- nen, i. d. R. handelt es sich bei dieser Erkrankungsgruppe aber um Systemerkrankungen. Neben der Manifestation an der Haut gibt es typische klinisch Manifestationsmuster an Organen mit entsprechenden Symptomen und Verläufen. Diese helfen in der Abgrenzung dieser Vaskulitiden von der kutanen IgM oder lgG Immunkomplex-Vaskulitis [2, 3, 4, 5, 6, 7, 9, 21, 43]. Das Befallsmuster der AAV reicht von den postkapillären Venolen bis hin zu kleinen Arterien. Häufig zeigt sich histologisch das Bild einer leukozytoklastischen Vaskulitis neben granulomatösen Entzündungsarealen [2, 3, 18]. Klinisch und im Verlauf besonders ähnlich ist die Drug-induced skinlimited AAV, hier kann bei der Abgrenzung zur kutanen IgM oder IgG Immunkomplex-Vaskulitis ein positiver ANCA Titer helfen [28, 39] ( Abb. 14a- Abb. 14b).

Ein weiterer Vertreter der Gruppe der „small vessel“ Vaskulitiden ist die Kryoglobulinämische Vaskulitis. Kryoglobuline sind Immunglobuline die bei $37,0^{\circ} \mathrm{C}$ präzipitieren. Es werden monoklonale (Typ I, IgM oder IgG) und gemischte (Typ-II-IgM und -III-IgM) Formen der Kryoglobulinämie unterschieden. Gemischt bedeutet, sie enthalten neben IgM weitere Proteine wie z. B. den Rheumafaktor. $80 \%$ der Erkrankungen, speziell der gemischten Formen, sind mit einer Hepatitis C-Infektion assoziiert [2, 3, 22, 23, 24]. Eine Vaskulitis mit entsprechender Hautmanifestation ist am häufigsten beim Typ II, aber auch Typ I und Typ III zeigen entsprechende Bilder. Klinisch beginnt diese Vaskulitis an der Haut mit den klassischen 

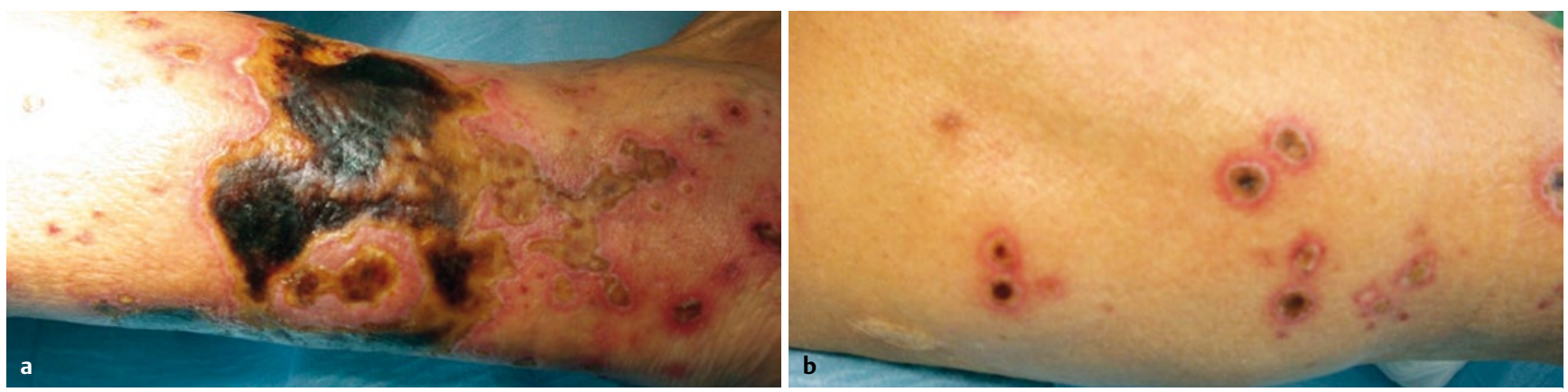

Abb. 14 Drug-associated immune complex vasculitis nach Einnahme von Arcoxia ${ }^{\circledR}$ (Etericoxib) (a lateraler Unterschenkel b über dem Kniegelenk)
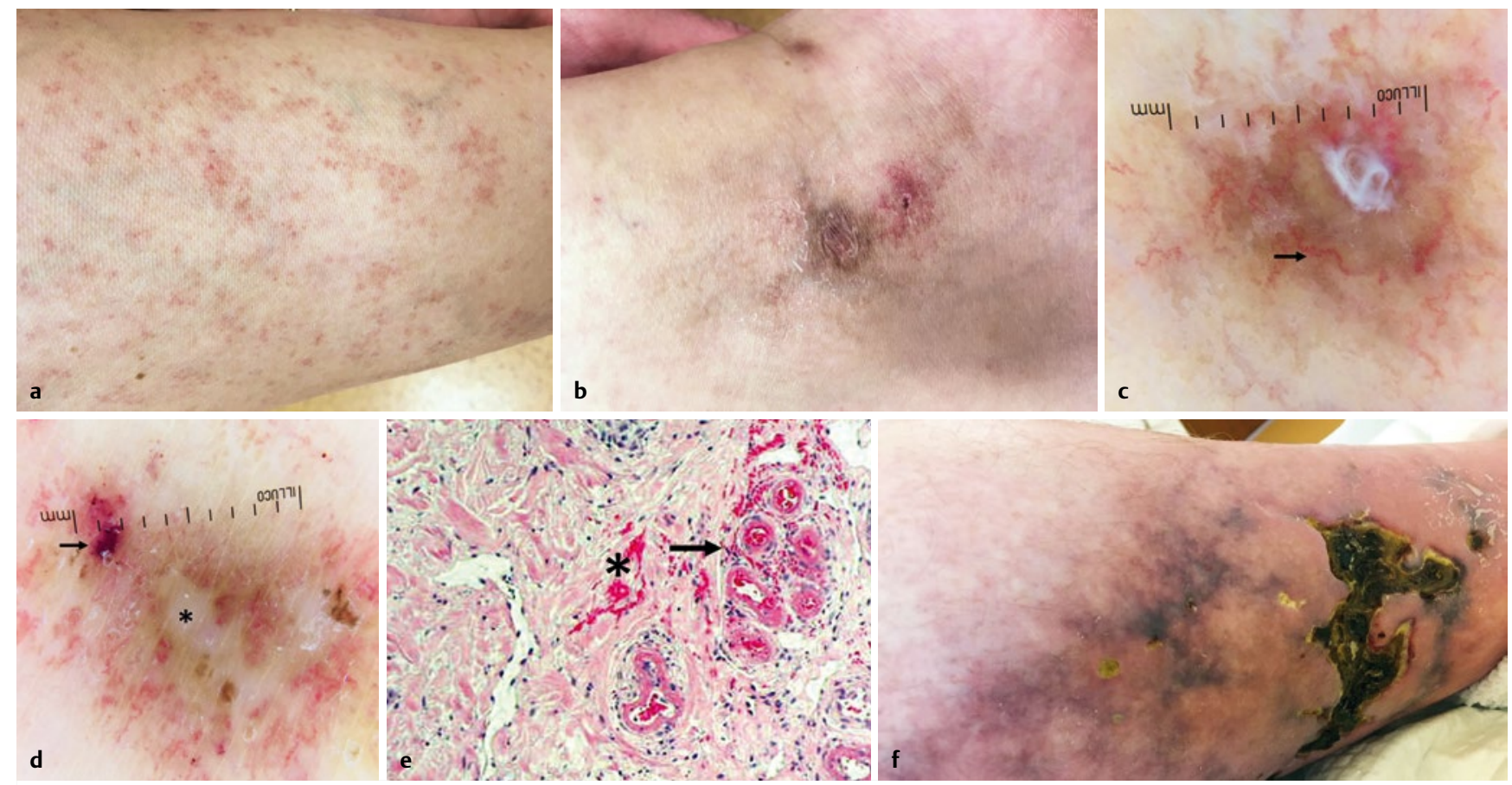

-Abb. 15 Antiphospholipid-Syndrom (IgA-Typ) mit klinisch petechial imponierenden Veränderungen an den Unterschenkeln mit kleinsten inflammatorisch wirkenden Ulzerationen neben hyperpigmentiert umrandeten Atrophie blanche Herden (a Überblick, b Detail); c Dermatoskopie Antiphospholipid-Syndrom IgA Subtyp: es kommt ein kleines Ulcus zur Darstellung mit aufgelagertem Creme-Rest umgeben von linearen korkenzieherartig konfigurierten Gefäßen (Pfeil); d Dermatoskopie Antiphospholipid-Syndrom IgA-Subtyp: es zeigen sich abgeheilte porzellanfarbene Areale $\left({ }^{*}\right.$ ), umgeben von Pigmentablagerungen und ektasierten, z. T. frisch thrombosierten Gefäßen (Pfeil); e Histologisches Bild einer Vaskulopathie: subepidermal zeigen sich vermehrt kleine Gefäße, gruppiert angeordnet (Pfeil) (entspricht dem klinischen Korrelat des korkenzieherartigen Musters) mit hyalinen Wandverdickungen ohne perivaskuläres Infiltrat, daneben bestehen Erythrozytenextravasate $\left({ }^{*}\right)$ (HE Färbung, $10 \times / 0,25)$; f Vollbild eines klinisch an der Haut manifesten Antiphospholipid-Syndroms mit einer hämorrhagisch livid-düsterroten Livedo-Zeichnung neben bizarr konfigurierten infarziert imponierenden Ulzera mit festhaftenden Nekrosen.

Effloreszenzen einer LcV, diese frühen Hautveränderungen werden jedoch klinisch selten wahrgenommen da sie rasch zu großen purpurischen Flächen konfluieren und in ausgedehnte Nekrosen übergehen. Diese zunächst wenig inflammatorisch imponierenden Hautveränderungen hinterlassen im Verlauf hochinflammatorisch imponierende Ulzerationen, mit welchen die Patienten dann häufig erstmalig vorstellig werden. Auch hier zeigen sich immunhistologisch perivaskuläre Ablagerung von IgM und C3, differentialdiagnostisch wegweisend sind hier die durch eosinophil-glasig imponierende Strukturen (= Kryoglobulin-Antikörper-Komplexe) verschlossenen Gefäße. Betroffen sind überwiegend postkapilläre Venolen, vereinzelt Arteriolen und mittelgroße Gefäße.

Eine weitere Erkrankungsgruppe, die oft primär petechial anmutende Veränderungen als eine Art prämonitorische Effloreszenz aufweist, bevor sich Nekrosen und Ulzerationen zeigen, sind zum einen das Antiphospholipid-Syndrom ( $\vee$ Abb. 15a- $>$ Abb. 15b, - Abb. 15f), (sowohl das primäre als auch das sekundäre im Rahmen eines Lupus erythematodes) und zum anderen die Livedovasculopathie ( $\vee$ Abb. 16a- $>$ Abb. 16b) als Vaskulopathien. Beide Erkrankungen können z. T. klinisch hochinflammatorische Ulzerationen aufweisen mit einem petechial anmutenden Begleitsaum und 

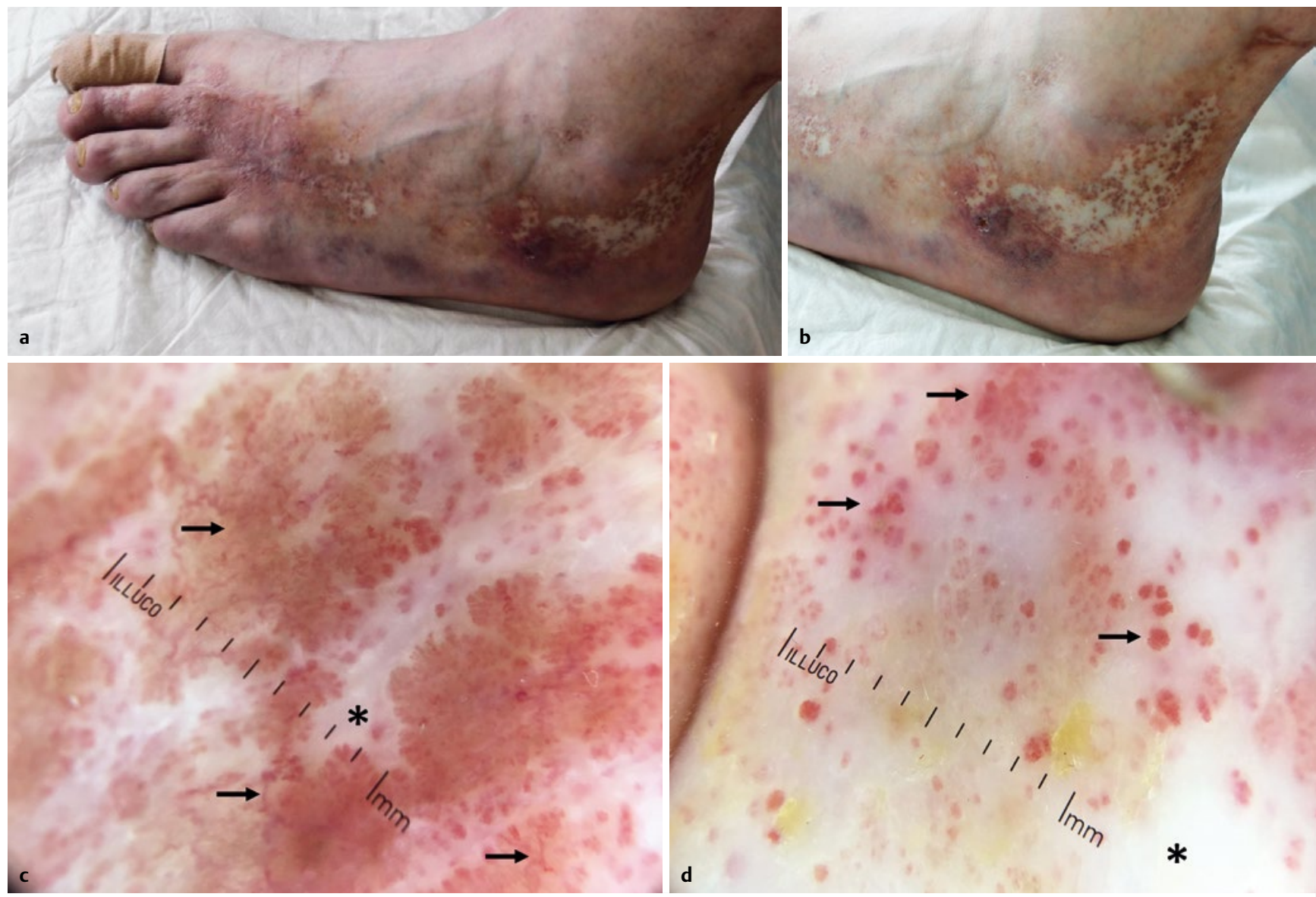

- Abb. 16 Klinisch Livedovaskulopathie mit ausgedehnten Atrophie blanche Herden neben kleinen sehr schmerzhaften Ulzerationen im VorfußKnöchelbereich und livid-erythematösen Arealen (a Übersicht; b Detail); c Dermatoskopie Livedovaskulopathie: zentral porzellan-farbene atrophe Areale $\left({ }^{*}\right.$ ), in der Umgebung zeigen sich einzelne teleangiektatische lineare Gefäße (Pfeile) neben überwiegend glomerulären Gefäßmustern. Es zeigen sich keine punktförmigen Gefäßmuster als Hinweis auf eine entzündliche Genese der Erkrankung; d Dermatoskopie Livedovaskulopathie: sowohl in den porzellan-farbenen atrophen Areale $\left({ }^{*}\right)$ als auch in der Umgebung zeigen sich disseminiert glomeruläre Gefäßmuster (Pfeile).

-muster. Dermatoskopisch zeigen sich bei beiden Erkrankungen die porzellanfarbene bizarr konfigurierte atrophe Areale begleitet bzw. umrandet von sowohl linearen, überwiegend korkenzieherartig konfigurierten, als auch glomerulären Gefäßmustern [50] ( Abb. 13b->Abb. 13d neu, $>$ Abb. 15c- $\rightarrow$ Abb. 15e, $>$ Abb. 14b, - Abb. 14c neu, > Abb. 16c->Abb. 16d). Klassisch entzündliche Veränderungen sind nicht sichtbar (Punktförmige Gefäßmuster), ebenso keine Hämorrhagien. Zusätzliche anamnestische (Thrombophilie), klinische (klassische Trias der Livedovasculopathie - sequentielles Auftreten einer Livedo racemosa, von sehr schmerzhaften Ulzerationen und einer Atrophie blanche) bzw. paraklinische Marker (Antiphospholipid-Antikörper, Histologie) machen eine Abgrenzung zu einer Vaskulitis möglich [30, 31, 32, 33, 34, $35,36,37,38]$.

Hinweisend auf einen vaskulitischen Prozess im Rahmen eines Ulcus mit Nekrosebildung ist der rasche Verlauf, die hochinflammatorischen, bizarr konfigurierten Ränder und die Schmerzhaftigkeit des Befundes. Häufig sind neben den Ulzerationen und Nekrosen in den Randbereichen die palpablen Petechien sichtbar. Sind diese zu detektieren sollte eine Vaskulitis als Ursache der Ulzeration erwogen werden.

\section{Therapie}

Das therapeutische Konzept orientiert sich in erster Linie an der Unterbrechung der Kausalkette des Erkrankungsbildes. Dies beinhaltet in den meisten Fällen das Absetzen möglicher auslösender Substanzen [21, 42, 44]. Neben neuen Medikamenten als solche sollten vor allem Medikament mit Dosis-Anpassungen 14 Tagen vor Auftreten der Hautveränderungen genauer betrachtet werden. Ebenso ist es erforderlich nach Begleiterkrankungen, die die Elimination von Medikamenten beeinflussen (z. B. Leber- und Niereninsuffizienz) bzw. interagierende Medikamente, die die Konzentration des auslösenden Medikamentes verändern.

Daneben ist die Therapie von entsprechenden erregerassoziierten Erkrankungen zielführend.

Neben der ursächlichen Behandlung stehen dann symptomatische Therapien wie Antihistaminika intern zur Reduktion der symptomvermittelnden Mediatoren zur Verfügung und in Fällen mit absehbar schwerem Verlauf (Ausbildung von hämorrhagischen Epidermolysen - Bullae - Nekrosen) auch der Einsatz von Kortikosteroiden u. a. mit dem Ziel der Reduktion der Zellgetragenen Inflammation. Lokale Maßnahmen mit steroidhaltigen Externa und eine komplexe Entstauung (Kompression, Lymphdrainagen) können den Verlauf positiv beeinflussen [2, 19, 21, 41, 42, 44]. 
Bei den medikamentenassoziierten Varianten der kutanen IgModer IgG-Immunkomplex-Vaskulitis sind Rezidive auf Grund eines Sensibilisierungsmechanismus möglich. Eine entsprechende Dokumentation und Information des Patienten und der weiterbehandelnden Ärzte sollte erfolgen.

Bei der 97-jährigen Patientin wurde das Torasemid abgesetzt und durch Diuretika einer anderen Stoffklasse ersetzt, zusätzlich wurde eine Akut-Hämofiltration (Dialyse) vorgenommen und Kortikosteroide eingesetzt. Darunter kam die Vaskulitis zum Stillstand, die Hautveränderungen kamen, wenn auch sehr zeitverzögert, im Verlauf zur Abheilung.

Auch im 2. Kasus kam die Vaskulitis nach primär komplettem Absetzen des Torasemids zum Stillstand. Im Verlauf wurde die Substanz jedoch erneut bei dringender kardialer Indikation vorsichtig eingeschlichen. Ab einer Dosis von $2 \times 10 \mathrm{mg}$ über 2 Tage zeigten sich neue vaskulitische Effloreszenzen ( $\boldsymbol{A} \boldsymbol{b} \boldsymbol{b}$. $3 \boldsymbol{3} \rightarrow-\boldsymbol{A} \boldsymbol{b} \boldsymbol{b} .3 \boldsymbol{d}$ ). Anamnestisch ließ sich im Nachgang an diesen beobachteten Aufdosierungseffekt ein ähnliches Ereignis 1 Jahr zurückliegend bei dem betreuenden Hausarzt erfragen. Bei Sichtung der Krankenunterlagen vom Erstereignis zeigt sich eine wahrscheinliche Auslösedosis von $2 \times 10 \mathrm{mg} / \mathrm{d}$ Torasemid, anschließend war die Substanz bis zum Zweitereignis konsequent abgesetzt gewesen ( $\boldsymbol{A} \boldsymbol{A b b}$. 3e). Anhand dieses 2. Falles lässt sich der reine Effekt des Erreichens der Äquivalenzzone ohne andere Einflussfaktoren anschaulich ableiten.

\section{Diskussion}

Bei der kutanen IgM oder lgG Immunkomplex-Vaskulitis handelt es sich um eine Vaskulitis der kleinen Gefäße, in der aktuell geltenden Klassifikation mit alleiniger Manifestation an der Haut ohne weitere Organmanifestation als „single organ vasculitis“ (SOV). Histologisch zeigt sie das Bild einer leukozytoklastischen Vaskulitis. Es handelt sich um eine typische Immunkomplex-Erkrankung deren Ursache am häufigsten medikamentös und infektassoziiert verankert ist. Das Krankheitsbild zeigt zwei typische aufeinander folgende klinische Verlaufsphasen, zunächst die Phase der palpablen Petechien, wenn nicht zu diesem Zeitpunkt gestoppt gefolgt von Epidermolysen in Form von Vesikula und Bullae und Nekrosen, die dann in hochinflammatorische Ulzerationen übergehen können. Dieser klinische Verlauf macht die Auswahl an Differentialdiagnosen vielfältig, umso wichtiger ist eine detaillierte Anamnese, eine genaue klinische Befunderfassung und die entsprechende Diagnostik. Therapeutisch liegt das Potential in der Ursachenklärung und wenn kausal wahrscheinlich dem Absetzen des auslösenden Agens oder der Therapie einer zugrundeliegenden Infektion. Die Erkrankung verläuft häufig auch ohne den Einsatz von systemischen Kortikosteroiden spontan. Die Prognose ist in der Regel gut, Rezidive sind möglich, aber selten.

\section{Literatur}

[1] Jennette JC et al. 2012 Revised International Chapel Hill Consensus Conference Nomenclature of Vasculitides, Arthritis \& Rheumatism 2013: 65: 1-11
[2] Sunderkötter C. Vaskulitis und Vaskulopathien, Braun-Falco's Dermatologie. Venerologie und Allergologie 2018; 3: 1-44. DOI: 10.1007/9783-662-49546-9_64-1

[3] Sunderkötter $\mathrm{CH}$, Zelger B, Chen KR et al.Nomenclature of Cutaneous Vasculitis Dermatologic Addendum to the 2012 Revised International Chapel Hill Consensus Conference Nomenclature of Vasculitides.

[4] Watts RA. Evolving concepts in classification of systemic vasculitis: where are we and what is the way forward? International Journal of Rheumatic Diseases, 2018

[5] StonejH. Kelley and Firestein's Textbook of Rheumatology (Tenth Edition), 2017, Classification and Epidemiology of Systemic Vasculitis

[6] Calabrese LH, Michel BA, Bloch DA et al. The American College of Rheumatology 1990 criteria for the classification of hypersensitivity vasculitis. Arthritis Rheum, 1990

[7] Carlson JA, Ng BT, Chen KR.Cutaneous vasculitis update: diagnostic criteria, classification, epidemiology, etiology, pathogenesis, evaluation and prognosis.Am J Dermatopathol 2005; 27: 504-528

[8] Khetan P, Sethuraman G, Khaitan BK et al. An aetiological \& clinicopathological study on cutaneous vasculitis. Indian J Med Res 2012; 135 : 107-113

[9] Sunderkötter C, Sindrilaru A. Classification of vasculitis. Europ J Dermatol 2006; 16(2): 114-24

[10] Kawasaki Y et al. Clinical and pathological features of children with Henoch-Schoenlein purpura nephritis: risk factors associated with poor prognosis. Clin Nephrol 2003; 60: 153-160

[11] Loricera J et al. Single-organ cutaneous small-vessel vasculitis according to the 2012 revised International Chapel Hill Consensus Conference Nomenclature of Vasculitides: a study of 60 patients from a series of 766 cutaneous vasculitis cases. Rheumatology 2015; 54: 77-82

[12] Ratzinger $G$ et al. Das Vaskulitis-Rad-ein algorithmischer Ansatz für kutane Vaskulitiden. JDDG 2015: 1092-1118

[13] King TC. Elsevier's Integrated Pathology, 2007, Inflammation, Inflammatory Mediators, and Immune-Mediated Disease

[14] Birdsall HH. Mandell, Douglas, and Bennett's Principles and Practice of Infectious Diseases (Eighth Edition), Adaptive Immunity, 2015

[15] Actor JK. Adaptive Immune Response and Hypersensitivity, in Elsevier's Integrated Review Immunology and Microbiology (Second Edition), 2012

[16] Mak TW, Saunders ME. Allergy and Hypersensitivity. The Immune Response, 2006

[17] Lentsch $A B$, Ward PA. Regulation of inflammatory vascular damage. J Pathol 2000

[18] Carlson JA.The histological assessment of cutaneous vasculitis. Histopathology 2010; 56: 3-23

[19] Stone JH, Nousari HC."Essential" cutaneous vasculitis: what every rheumatologist should know about vasculitis of the skin.Curr Opin Rheumatol 2001; 13: 23-34

[20] Jessop S]. Cutaneous leucocytoclastic vasculitis: a clinical and aetiological study. Br J Rheumatol 1995

[21] Sunderkötter C, Roth J, Bonsmann G. Leukozytoklastische Vaskulitis. Hautarzt 2004; 55(8): 759-785

[22] Brouet et al. Kryoglobulinämie. Am J Med 1974; 57: 775-788

[23] Staak JO, Glossmann JP, Diehl et al. Hepatitis-C-Virus-assoziierte Kryoglobulinämie - Pathogenese, Diagnostik und Therapie. Medizinische Klinik October 2002; 97: 601-608

[24] Pischke S, Cornberg M, Manns MP. Hepatitis-assoziierte Kryoglobulinämie. Der Internist, 2008

[25] Davin JC. Henoch-Schonlein purpura nephritis. Pathophysiology, treatment, and future strategy. Clinical journal of the American Society of Nephrology 2011; 6: 679-689. DOI: 10.2215/CJN.06710810 
[26] Blanco R, Martinez-Taboada VM, Rodriguez-Valverde V et al. Cutaneous vasculitis in children and adults. Associated diseases and etiologic factors in 303 patients. Medicine (Baltimore), 1998

[27] Blanco R, Martinez-Taboada VM, Rodriguez-Valverde V et al. HenochSchonlein purpura in adulthood and childhood: two different expressions of the same syndrome. Arthritis Rheum, 1997

[28] Calabrese LH, Duna GF. Drug-induced vasculitis. Curr Opin Rheumatol 1996; 8: 34-40

[29] Michel BA, Hunder GG, Bloch DA. Hypersensitivity vasculitis and Henoch- Schonlein purpura: a comparison between the 2 disorders. J Rheumatol, 1992

[30] Cervera R. Antiphospholipid sydrome. In: Thrombosis Research 2017; 151: 43-47. DOI: 10.2016/S0049-3848(17)30066-X

[31] Gómez-Puerta JA, Cervera R. Diagnosis and classification of the antiphospholipid syndrome. Journal of autoimmunity 2014; 48-49: 20-25. DOI:10.1016/j.jaut.2014.01.006

[32] Cervera R. Antiphospholipid syndrome. Thrombosis Research 2017; 151:43-47. DOI: 10.1016/S0049-3848(17)30066-X

[33] Gómez-Puerta JA, Cervera R. Diagnosis and classification of the antiphospholipid syndrome. Journal of autoimmunity 2014; 48-49: 20-25. DOI: 10.1016/j.jaut.2014.01.006

[34] Miyakis S, Lockshin MD, Atsumi T et al. International consensus statement on an update of the classification criteria for definite antiphospholipid syndrome (APS). J Thromb Haemost 2006; 4: 295- 306

[35] Kerk N, Goerge T. Livedoid vasculopathy - a thrombotic disease. VASA 2013; 42: 317-322

[36] Alavi A, Hafner J, Dutz JP et al. Livedoid vasculopathy: an in-depth analysis using a modified Delphi approach. J Am Acad Dermatol 2013; 69: $1033-1042$

[37] Jorizzo JL. Livedoid vasculopathy: what is it? Arch Dermatol 1998; 134 : 491-493
[38] Ahmed M, Lutze S, Herrmann A et al. Livedovaskulopathie - eine Erkrankung seltener Blutgruppenmerkmale? vasomed 2017; 29: 223224

[39] Holder SM, Joy MS, Falk RJ. Cutaneous and systemic manifestations of drug-induced vasculitis. Ann Pharmacother 2002

[40] Hengge UR et al. Purpura fulminans. A fatal consequence of a widely used medication? Hautarzt 2002; 53: 483-487

[41] Chen KR, Carlson JA. Clinical approach to cutaneous vasculitis. Am J Clin Dermatol 2008; 9: 71-92

[42] Carlson JA, Cavaliere LF, Grant-Kels JM. Cutaneous vasculitis: diagnosis and management. Clin Dermatol 2006; 24: 414-429

[43] Carlson JA, Chen KR.Cutaneous vasculitis update: small vessel neutrophilic vasculitis syndromes. Am J Dermatopathol 2006; 28: 486-506

[44] Sunderkötter C, Bonsmann G, Sindrilaru A et al. Management of leukocytoclastic vasculitis. J Dermatolog Treat 2005

[45] Averbeck M, Gebhardt C, Emmrich F et al. Simon Immunologische Grundlagen der Allergien, JDDG 2007; 5:1015-1028

[46] Ashfaq A, Marghoob MD, Malvehy J et al. An Atlas of Dermoscopy, Second Edition (Englisch). Taschenbuch - 26. Juli 2012

[47] Trautmann A, Kleine-Tebbe J. Allergologie in Klinik und Praxis: Allergene - Diagnostik - Therapie 25. Oktober 2017

[48] Vázquez-López F, FueyoA, Sánchez-Martín J et al. Dermoscopy for the Screening of Common Urticaria and Urticaria Vasculitis, Arch Dermatol 2008; 144: 568. doi:10.1001/archderm.144.4.568

[49] Ashfaq AM, Alon S, Vazquez-Lopez F et al. Dermoscopic Patterns of Purpuric Lesions. Arch Dermatol 2010; 146: 938. doi:10.1001/archdermatol.2010.162

[50] Chu-Sung Hu S, Chen GS, Lin CL et al. Dermoscopic features of livedoid vasculopathy, Medicine (Baltimore) 2017; 96: e6284 